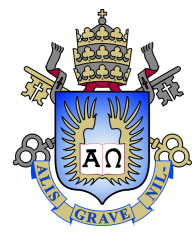

Aline de Melo Machado

\title{
Limit Theorems for Uniquely Ergodic Systems
}

Dissertation presented to the Programa de Pós-Graduação em Matemática of PUC-Rio in partial fulfillment of the requirements for the degree of Mestre em Matemática.

Advisor: Prof. Silvius Klein 


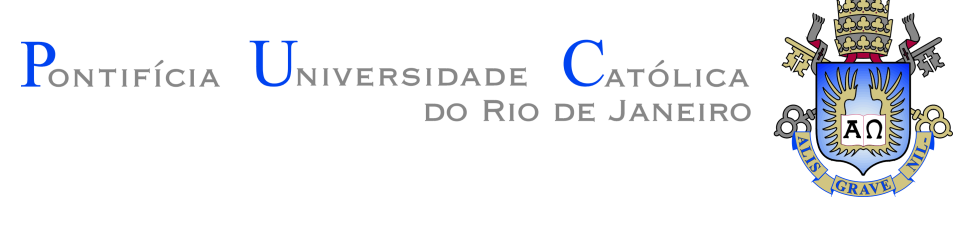

Aline de Melo Machado

\section{Limit Theorems for Uniquely Ergodic Systems}

Dissertation presented to the Programa de Pós-Graduação em Matemática of PUC-Rio in partial fulfillment of the requirements for the degree of Mestre em Matemática. Approved by the undersigned Examination Committee.

Prof. Silvius Klein

Advisor

Departamento de Matemática - PUC-Rio

Prof. Simon Griffiths

Departamento de Matemática - PUC-Rio

Prof. Bruno Rodrigues Santiago

Instituto de Matemática e Estatística - UFF

Profa. Yuri Ki

Instituto de Matemática e Estatística - UFF

Prof. Márcio da Silveira Carvalho

Vice Dean of Graduate Studies

Centro Técnico Científico - PUC-Rio

Rio de Janeiro, September 20th, 2018 
All rights reserved.

\section{Aline de Melo Machado}

Has a Bachelor degree in Mathematics from Universidade Federal Fluminense, Volta Redonda-RJ, Brasil.

Bibliographic data

De Melo Machado, Aline

Limit Theorems for Uniquely Ergodic Systems / Aline de Melo Machado; advisor: Silvius Klein. - Rio de janeiro: PUCRio, Departamento de Matemática, 2018.

v., 79 f: il. color. ; $30 \mathrm{~cm}$

Dissertação (mestrado) - Pontifícia Universidade Católica do Rio de Janeiro, Departamento de Matemática.

Inclui bibliografia

1. Matemática - Dissertação. 2. Sistemas Dinâmicos Unicamente Ergódicos;. 3. Teoremas Ergódicos;. 4. Cociclo linear;. 5. Expoente de Lyapunov;. 6. Taxa de convergência de médias de Birkhoff.. I. Klein, Silvius. II. Pontifícia Universidade Católica do Rio de Janeiro. Departamento de Matemática. III. Título. 
To my parents, for their support and encouragement. 


\section{Acknowledgments}

I would like to thank my parents José Cláudio and Adriana and my sister Julia for the great support and encouragement, which were fundamental for my conclusion of the master's degree.

I would like to thank my advisor Prof. Silvius Klein for the continuous support, for his patience, motivation, and immense knowledge.

Besides my advisor, I would like to thank the members of the thesis committee, Bruno Santiago, Simon Griffiths and Yuri Ki, for accepting the invitation and for the several useful comments that helped significantly in the text of this dissertation.

I would like to thank Xiao-Chuan Liu for his useful suggestions.

My sincere thanks also goes to Creuza and all administrative staff of the Department of Mathematics for helping me to fulfill all the bureaucracy requirements.

I thank all the friends of PUC-Rio for the relaxed moments inside and outside of the university, especially with some friends of room 850 and 858. In particular, I thank João Marcos and Lucas, who accompanied me in some disciplines and for the help during these courses.

I dedicate this work and give special thanks to my favorite person who helped me a lot during the master's degree.

This study was financed in part by the Coordenação de Aperfeiçoamento de Pessoal de Nível Superior - Brasil (CAPES) - Finance Code 001. 


\section{Abstract}

De Melo Machado, Aline; Klein, Silvius (Advisor). Limit Theorems for Uniquely Ergodic Systems. Rio de Janeiro, 2018. 79p. Dissertação de Mestrado - Departamento de Matemática, Pontifícia Universidade Católica do Rio de Janeiro.

The fundamental results in ergodic theory - the Birkhoff theorem and the Kingman theorem - refer to the almost everywhere convergence of additive and respectively subadditive ergodic processes. It is well known that given a uniquely ergodic system and a continuous observable, the corresponding Birkhoff averages converge everywhere and uniformly. It is therefore natural to ask what happens with Kingman's theorem when the system is uniquely ergodic. The first objective of this dissertation is to answer this question following the work of A. Furman. Moreover, we present some extensions and applications of this result for linear cocycles, which were obtained by S. Jitomirskaya and R. Mavi. Our second objective is to prove a new result regarding the rate of convergence of the Birkhoff averages for a certain type of uniquely ergodic process: a Diophantine torus translation with Hölder continuous observable.

\section{Keywords}

Uniquely Ergodic Dynamical Systems; The ergodic theorems; Linear cocycle; Lyapunov exponents; Rate of convergence of Birkhoff averages. 


\section{Resumo}

De Melo Machado, Aline; Klein, Silvius. Teoremas Limite para

Sistemas Unicamente Ergódicos. Rio de Janeiro, 2018. 79p. Dissertação de Mestrado - Departamento de Matemática, Pontifícia Universidade Católica do Rio de Janeiro.

Os resultados fundamentais da teoria ergódica - o teorema de Birkhoff e o teorema de Kingman - se referem a convergência em quase todo ponto de um processo ergódico aditivo e subaditivo, respectivamente. É bem conhecido que dado um sistema unicamente ergódico e um observável contínuo, as médias de Birkhoff correspondentes convergem em todo ponto e uniformemente. Desta forma, é natural também se perguntar o que acontece com o teorema de Kingman quando o sistema é unicamente ergódico. O primeiro objetivo desta dissertação é responder a essa pergunta utilizando o trabalho de A. Furman. Mais ainda, apresentamos algumas extensões e aplicações desse resultado para cociclos lineares, que foram obtidas por S. Jitomirskaya e R. Mavi. Nosso segundo objetivo é provar um novo resultado sobre taxas de convergências de médias de Birkhoff, para um certo tipo de processo unicamente ergódico: uma translação diofantina no toro com um observável Hölder contínuo.

\section{Palavras-chave}

Sistemas Dinâmicos Unicamente Ergódicos; Teoremas Ergódicos; Cociclo linear; Expoente de Lyapunov; Taxa de convergência de médias de Birkhoff. 


\section{Table of contents}

1 Introduction $\quad 10$

2 Basic Concepts in Ergodic Theory $\quad 14$

2.1 Measure Preserving Dynamical System 14

2.2 Ergodic Systems 22

2.3 The Pointwise and Subadditive Ergodic Theorems 28

2.4 Uniquely Ergodic Systems 37

3 Furman's Theorem $\quad 42$

3.1 Preliminaries 42

3.2 On the subadditive ergodic theorem for uniquely ergodic systems 44

3.3 Uniform upper semicontinuity of the Lyapunov exponent 49

4 A convergence rate for Birkhoff sums of Diophantine torus translations $\quad \mathbf{5 4}$

4.1 Introduction and statement 54

4.2 Continued fractions $\quad 56$

$\begin{array}{ll}\text { 4.3 The Diophantine condition } & 62\end{array}$

4.4 Convergence rate of Birkhoff sums: idea of the proof 66

4.5 Convergence rate of Birkhoff sums: formal proof 69

$\begin{array}{lll}4.6 & \text { Related problems } & 76\end{array}$

$\begin{array}{ll}\text { Bibliography } & 78\end{array}$ 


\section{List of figures}

$\begin{array}{lll}\text { Figure } 1.1 & \text { Poincaré Recurrence } & 11\end{array}$

$\begin{array}{lll}\text { Figure 2.1 The trajectory of } z=e^{2 \pi i \omega} & 15\end{array}$

Figure 4.1 Theorem 4.2.4 illustration 62

$\begin{array}{lll}\text { Figure } 4.2 & \text { Illustration of the proof } & 69\end{array}$ 


\section{1}

\section{Introduction}

A dynamical system consists of a space $X$ and a rule that determines how points in $X$ evolve in time. Time can be either continuous (e.g. indexed by $\mathbb{R}$ ) or discrete (e.g. indexed by $\mathbb{Z})$.

In this work, we only consider the case of discrete time. Thus, the law of our system is a single map $T: X \rightarrow X$ and, given $x \in X$ we can think of $T^{n}(x)$ as representing where $x$ has moved to after time $n \in \mathbb{N}$.

We call the sequence

$$
x, T(x), T^{2}(x), \ldots, T^{n}(x), \ldots
$$

the orbit of $x$. If $T$ is an invertible map, then the sequence above is called the forward orbit of $x$.

One of the objectives of the area of dynamical systems is to study the behavior of these orbits. In general it is difficult or impossible to understand the behavior of every orbit. Instead, we could consider almost every orbit, with respect to an appropriate measure $\mu$ on the system. Such a measure is a T-invariant probability measure, that is, $\mu(X)=1$ and

$$
\mu(E)=\mu\left(T^{-1}(E)\right), \quad \text { for every measurable subset } E \subset X .
$$

A measurable subset $E$ of $X$ is said to be $T$-invariant if $E=T^{-1}(E)$. Heuristically, we say that $(X, \mu, T)$ is an ergodic system if the system is irreducible, in the sense that any $T$-invariant subset of $X$ has zero measure or total measure. We call the triple $(X, \mu, T)$ a uniquely ergodic system if $\mu$ is the only $T$-invariant probability measure on $X$.

Since understanding the behavior of orbits may be a very complex problem, we can use an observable ${ }^{1}$ function to still derive some characteristics of the system.

Ergodic theory has many applications in other areas of Mathematics

\footnotetext{
${ }^{1}$ a measurable function which is absolutely integrable.
} 
and Physics. For example, in Physics, we can use methods of ergodic theory in statistical mechanics to study the long-term average behavior of complex systems, such as the interactions of vibrating atoms in a crystal or the behavior of molecules in a gas. Moreover, we can apply the results of ergodic theory in many other areas of Mathematics. In geometry, it is very useful for studying geodesic flows on Riemannian manifolds. There are also applications in probability theory (for example, the theory of Markov chains). Furthermore, ergodic theory has connections with other fields such as harmonic analysis and number theory.

One of the first results in ergodic theory is the Poincaré recurrence theorem, which states that almost all points in any subset of the total space eventually revisit the set. Henri Poincaré discussed this theorem in 1890 [1] and Constantin Carathéodory proved it using measure theory in 1919 [2].

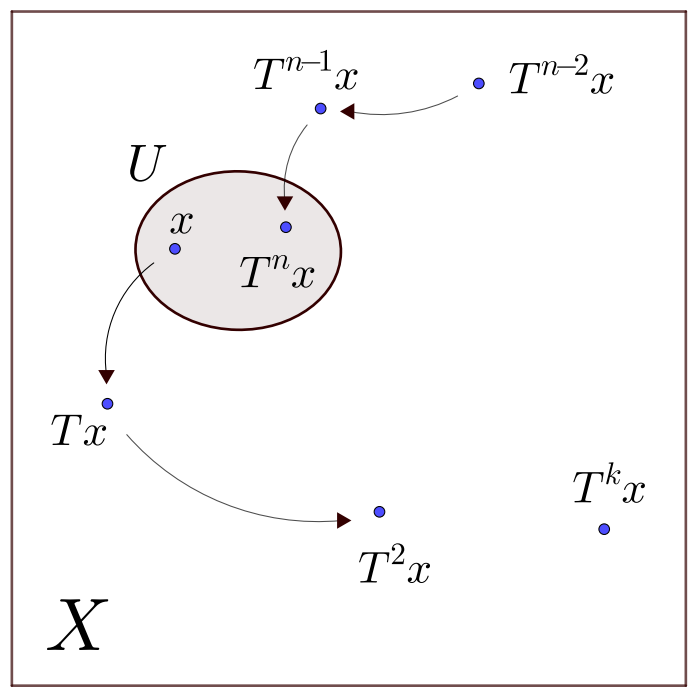

Figure 1.1: Poincaré Recurrence

In 1931, Birkhoff proved one of the most important theorems in ergodic theory [3]. Namely, given an ergodic system, the time average of a function along the trajectories converges almost everywhere to the space average. This result is known as the pointwise ergodic theorem. Moreover, for a uniquely ergodic system and a continuous observable, the corresponding Birkhoff averages converges everywhere and uniformly (see [4]).

A subadditive process on $(X, \mu, T)$ is a sequence $\left\{f_{n}\right\}_{n \in \mathbb{N}}$ of a measurable functions such that

$$
f_{n+m} \leq f_{n}+f_{m} \circ T^{n} \quad \text { for all } n, m \in \mathbb{N} \text {. }
$$


In 1968, Kingman established his subadditive ergodic theorem [5], that asserts the almost everywhere convergence of subadditive ergodic processes. That is, given any subadditive sequence $\left\{f_{n}\right\}_{n \in \mathbb{N}}$ in $L^{1}(X)$ (relative to the map $T: X \rightarrow X)$ the sequence $\left\{\frac{f_{n}}{n}(x)\right\}_{n \in \mathbb{N}}$ converges to a constant $L(f)$, for almost every $x \in X$. The constant $L(f)$ is called the Lyapunov exponent of the subadditive process $\left\{f_{n}\right\}_{n \in \mathbb{N}}$.

It is natural to ask what happens with Kingman's theorem when the system is uniquely ergodic. More precisely, it would be interesting to know if we have uniform convergence in Kingman's Theorem when we consider a uniquely ergodic system, as in the case of Birkhoff's Theorem.

The first objective of this dissertation is to describe the work of Furman [6], where this question was answered. ${ }^{2}$

Theorem 1.0.1 (Furman, 1997). Let $(X, \mu, T)$ be a compact, uniquely ergodic system and let $\left\{f_{n}\right\}_{n \in \mathbb{N}}$ be a subadditive sequence of continuous functions on $X$. Then, for every $x \in X$

$$
\limsup _{n \rightarrow \infty} \frac{1}{n} f_{n}(x) \leq L(f)
$$

However, for any $F_{\sigma}$ set $E$ (that is, $E$ is a countable union of closed subsets) with $\mu(E)=0$, there exists a continuous subadditive sequence $\left\{f_{n}\right\}$, such that

$$
\limsup \frac{1}{n} f_{n}(x)<L(f)
$$

We also present some extensions and applications of this result to linear cocycles. They were obtained by S. Jitomirskaya and R. Mavi [7].

Given an ergodic system $(X, \mu, T)$ and an observable $\varphi: X \rightarrow \mathbb{R}$, we denote by

$$
S_{n} \varphi(x):=\sum_{j=0}^{n-1} \varphi\left(T^{j} x\right)
$$

the corresponding Birkhoff sums. We recall that the Birkhoff average $\frac{1}{n} S_{n} \varphi(x)$ converges for almost every $x \in X$ to the space average $\int_{X} \varphi d \mu$.

Our second objective is to obtain an estimate on the convergence rate of the Birkhoff averages for certain types of uniquely ergodic systems and observables. More precisely, we consider a Diophantine torus translation with a Hölder continuous observable.

\footnotetext{
${ }^{2}$ This question was posed by Furstenberg.
} 
Let $\mathbb{T}=\mathbb{R} / \mathbb{Z}$ be the one dimensional torus endowed with the Lebesgue measure and let $T: \mathbb{T} \rightarrow \mathbb{T}, T x=x+\omega$ be the translation on $\mathbb{T}$ by an irrational frequency $\omega$, which satisfies a generic Diophantine condition. That is,

$$
\operatorname{dist}(k \omega, \mathbb{Z}) \geq \frac{c}{|k|(\log |k|)^{2}}
$$

for some $c>0$ and for all $k \in \mathbb{Z} \backslash\{0\}$.

We obtain the follow result.

Theorem 1.0.2 (S. Klein and A. Melo). Let $\varphi: \mathbb{T} \rightarrow \mathbb{R}$ be an observable and let $\omega \in \mathbb{T}$ be an irrational frequency. Assume that $\varphi$ is $\alpha$-Hölder continuous, for some $\alpha \in(0,1)$, and that $\omega$ satisfies the above Diophantine condition. Then there exists a universal constant $K$ such that for all $R \geq 1$ and $x \in \mathbb{T}$,

$$
\left|\frac{1}{R} \sum_{j=0}^{R-1} \varphi(x+j \omega)-\int_{\mathbb{T}} \varphi(x) d x\right| \leq \frac{K}{c} \cdot \frac{(\log R)^{3}}{R^{\frac{\alpha}{\alpha+1}}}\|\varphi\|_{\infty} .
$$

In a future work we will consider the same problem for any continuous observable (instead of Hölder); furthermore, we will also consider other types of transformations (e.g. the higher dimensional torus translation and the skewtranslation).

The rest of this dissertation is organized in three chapters as follows.

In chapter 2 we review basic notions in ergodic theory, such as the concepts and examples of measure preserving dynamical system, ergodic systems and uniquely ergodic systems. We also present in this chapter some important theorems in ergodic theory: Birkhoff's Ergodic Theorem and Kingman's Ergodic Theorem.

In Chapter 3, we present the proof of Furman's result. We then describe several consequences of this theorem.

In Chapter 4 we describe some basic results about continued fractions and the Diophantine condition. Then we present the proof of Theorem 4.1.1 on the rate of convergence of the Birkhoff averages for a Diophantine torus translation with Hölder continuous observable. 


\section{2}

\section{Basic Concepts in Ergodic Theory}

In this chapter we introduce the basic concepts in ergodic theory which will be needed in the next chapters. We define the notions of measure preserving dynamical system (Section 2.1), ergodic system (Section 2.2) and uniquely ergodic system (Section 2.4). For each of these concepts, we provide some examples.

We also present some important theorems in ergodic theory, such as the Birkhoff's Ergodic Theorem and the Kingman's Theorem (Section 2.3).

\section{1}

\section{Measure Preserving Dynamical System}

We define below the concept of measure space.

Definition 2.1.1. A measure space is a triplet $(X, \mathcal{B}, \mu)$ where

1. $X$ is a set, which we refer to as the phase space.

2. $\mathcal{B}$ is a $\sigma$-algebra, that is, $\mathcal{B}$ is a collection of subsets of $\mathrm{X}$ which contains the empty set, and which is closed under complements and countable unions. The elements of $\mathcal{B}$ are called measurable sets.

3. $\mu: \mathcal{B} \rightarrow[0,+\infty]$ is a $\sigma$-additive function, that is, if $E_{1}, E_{2} \ldots \in \mathcal{B}$ are pairwise disjoint then $\mu\left(\bigcup_{i=1}^{\infty} E_{i}\right)=\sum_{i=1}^{\infty} \mu\left(E_{i}\right)$. The function $\mu$ is called the measure.

If $\mu(X)=1$, then we say that $\mu$ is a probability measure and $(X, \mathcal{B}, \mu)$ is a probability space.

Definition 2.1.2. Let $(X, \mathcal{B})$ and $(Y, \mathcal{C})$ be measurable spaces. The function $T: X \rightarrow Y$ is said to be measurable if $T^{-1}(C) \in \mathcal{B}, \forall C \in \mathcal{C}$.

Let $(M, \mathcal{B}, \mu)$ be a measure space and $T: M \rightarrow M$ be a measurable function. 
Definition 2.1.3. We say that $\mu$ is a $T$-invariant measure on $M$, or that $T$ preserves $\mu$, if $\mu\left(T^{-1} E\right)=\mu(E)$, for all measurable subsets $E \subset M$.

Proposition 2.1.1. Let $T: M \rightarrow M$ be a measurable function and let $\mu$ be a measure on $M$. Then, $T$ preserves $\mu$ if, and only if,

$$
\int \phi d \mu=\int \phi \circ T d \mu
$$

for every $\mu$-integrable function $\phi: M \rightarrow \mathbb{R}$.

Definition 2.1.4. Let $T: M \rightarrow M$ be a measurable function. We say that $(M, \mathcal{B}, \mu, T)$ is a measure preserving dynamical system if $\mu$ is a $T$-invariant probability measure.

We present below examples of measure preserving dynamical system.

Example 2.1.1 (Translation on the unidimensional torus). Consider the unit circle $S^{1}=\left\{(x, y): x^{2}+y^{2}=1\right\} \subset \mathbb{R}^{2}$. We may also identify the unit circle in the real plane as a subset of the complex plane. More precisely,

$$
S^{1}=\{z \in \mathbb{C}:|z|=1\}=\left\{e^{2 \pi i \theta}: 0 \leq \theta<1\right\} \subset \mathbb{C} .
$$

Let $\theta$ be a real number, the transformation

$$
R_{\alpha}: S^{1} \rightarrow S^{1}, \quad e^{2 \pi i \theta} \mapsto e^{2 \pi i(\theta+\alpha)}=e^{2 \pi i \theta} \cdot e^{2 \pi i \alpha}
$$

is called the rotation map of $S^{1}$ by $2 \pi \alpha$.

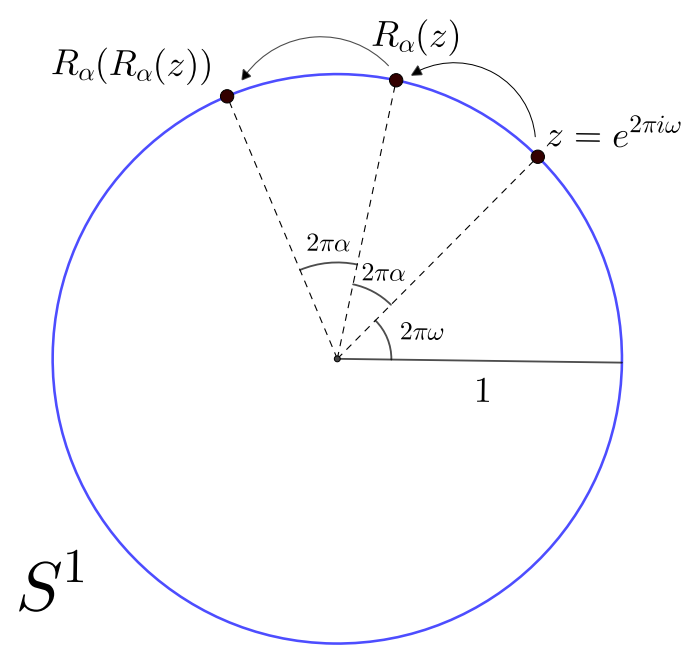

Figure 2.1: The trajectory of $z=e^{2 \pi i \omega}$

It is easy to see that $R_{0}$ is the identity map and $R_{\alpha} \circ R_{\tau}=R_{\alpha+\tau}$, for all $\alpha, \tau \in \mathbb{R}$. In particular, every rotation map $R_{\alpha}$ is invertible and $\left(R_{\alpha}\right)^{-1}=R_{-\alpha}$. 
We define a metric $d$ on the unite circle $S^{1}$ such as

$$
d\left(e^{2 \pi i \theta_{1}}, e^{2 \pi i \theta_{1}}\right)=\left\{\begin{array}{lll}
\left|\theta_{1}-\theta_{2}\right|, & \text { if } & \left|\theta_{1}-\theta_{2}\right| \leq \frac{1}{2} \\
1-\left|\theta_{1}-\theta_{2}\right|, & \text { if } & \frac{1}{2}<\left|\theta_{1}-\theta_{2}\right|<1
\end{array}\right.
$$

Hence, the rotation $R_{\alpha}$ is an isometry, that is, $d\left(R_{\alpha}\left(z_{1}\right), R_{\alpha}\left(z_{2}\right)\right)=d\left(z_{1}, z_{2}\right)$ for every $z_{1}, z_{2} \in S^{1}$.

There is an equivalent system to the rotation on the unit circle $S^{1}$.

Define the following equivalence relation $\sim$ on the real line $\mathbb{R}$ :

$$
x \sim y \Leftrightarrow x-y \in \mathbb{Z} .
$$

We use the notation $[x] \in \mathbb{R} / \mathbb{Z}$ to denote the equivalence class of any $x \in \mathbb{R}$. Moreover, the space $\mathbb{T}=\mathbb{R} / \mathbb{Z}$ formed by the equivalence classes is called the 1-dimensional torus. That is,

$$
\mathbb{R} / \mathbb{Z}=\{x+\mathbb{Z}: x \in[0,1)\}
$$

then $\mathbb{R} / \mathbb{Z}=[0,1] / 0 \sim 1$.

The map $\psi: \mathbb{R} / \mathbb{Z} \rightarrow S^{1}$ given by

$$
x \mapsto \psi(x)=e^{2 \pi i x}
$$

establishes a one-to-one correspondence between $\mathbb{R} / \mathbb{Z}$ and $S^{1}$. The distance $d$ defined in (2.1), gives the following distance on $\mathbb{R} / \mathbb{Z}$, that we also denote with the same symbol $d$ :

$$
d(x, y)=d(x-y, \mathbb{Z})=\min \{|x-y-k|: k \in \mathbb{Z}\})
$$

for every $x, y \in \mathbb{R} / \mathbb{Z}$.

Moreover, given $\alpha \in \mathbb{R}$, we may also define $T_{\alpha}: \mathbb{T} \rightarrow \mathbb{R}$

$$
T_{\alpha}(x)=x+\alpha \bmod 1
$$

which is called the translation map of $\mathbb{T}$ by $\alpha$.

Since $\psi$ is an isometric and bijective map between $S^{1}$ and $\mathbb{R} / \mathbb{Z}$, it follows that the systems $\left(S^{1}, d\right)$ and $(\mathbb{T}, d)$ are topologically conjugated. Hence, from now on, we only consider the translation map $T_{\alpha}$ on the torus $\mathbb{T}$.

Define $\pi: \mathbb{R} \rightarrow \mathbb{T}$ to be the canonical projection such that $\pi(x)=[x]$. We say that a set $E \subset \mathbb{T}$ is measurable if $\pi^{-1}(E)$ is a measurable subset of 
the real line. Let $m$ be the Lebesgue measure on the real line. We define the Lebesgue measure $\mu$ on the torus to be given by

$$
\mu(E)=m\left(\pi^{-1}(E) \cap[k, k+1)\right), \quad \forall k \in \mathbb{Z}
$$

It is clear that $\mu$ is a probability measure.

Proposition 2.1.2. Let $\mu$ be the measure defined above. Then, $\mu$ is invariant under every translation.

Proof. Let $k$ be the integer part of $\theta$. First, note that

$$
\pi^{-1}(E) \cap[k+1, \theta+1)=\left(\pi^{-1}(E) \cap[k, \theta)\right)+1 .
$$

Since $m$ is invariant under all the translation maps,

$$
\begin{aligned}
m\left(\left(\pi^{-1}(E)-\theta\right) \cap[0,1)\right) & =m\left(\left(\pi^{-1}(E) \cap[\theta, \theta+1)\right)\right. \\
& =m\left(\left(\pi^{-1}(E) \cap[\theta, k+1)\right)+m\left(\left(\pi^{-1}(E) \cap[k+1, \theta+1)\right)\right.\right. \\
& =m\left(\left(\pi^{-1}(E) \cap[\theta, k+1)\right)+m\left(\pi^{-1}(E) \cap[k, \theta)\right)\right. \\
& =m\left(\left(\pi^{-1}(E) \cap[k, k+1)\right) .\right.
\end{aligned}
$$

On the other hand, by definition, $\pi^{-1}\left(T_{\theta}^{-1}(E)\right)=\pi^{-1}(E)-\theta$ for all measurable subset $E \subset S^{1}$. Then, we have that

$$
\begin{aligned}
\mu\left(T_{\theta}^{-1}(E)\right) & =m\left(\pi^{-1}\left(T_{\theta}^{-1}(E) \cap[0,1)\right)\right)=m\left(\left(\pi^{-1}(E)-\theta\right) \cap[0,1)\right) \\
& =m\left(\left(\pi^{-1}(E) \cap[k, k+1)\right)\right. \\
& =\mu(E)
\end{aligned}
$$

for every measurable subset $E \subset S^{1}$.

Proposition 2.1.2 shows that $\left(\mathbb{T}, \mu, T_{\alpha}\right)$ is a measure preserving dynamical system, for any real number $\alpha$.

Example 2.1.2 (Translation on the higher dimensional torus). As in example 2.1.1, we can define a space of equivalence classes in $\mathbb{R}^{d}$.

For each $d \geq 1$, consider the equivalence relation on $\mathbb{R}^{d}$, given by

$$
\left(x_{1}, \ldots, x_{d}\right) \sim\left(y_{1}, \ldots, y_{d}\right) \Leftrightarrow\left(x_{1}-y_{1}, \ldots, x_{d}-y_{d}\right) \in \mathbb{Z}^{d}
$$


We will use the notation $[x]$ or $\left[\left(x_{1}, \ldots, x_{d}\right)\right]$ to denote the equivalence class of any $x=\left(x_{1}, \ldots, x_{d}\right) \in \mathbb{R}^{d}$. Moreover, the space

$$
\mathbb{T}^{d}=\mathbb{R}^{d} / \mathbb{Z}^{d}=(\mathbb{R} / \mathbb{Z})^{d}
$$

formed by the equivalence classes, is called the d-dimensional torus or, simply, the d-torus.

Given $\theta=\left(\theta_{1}, \ldots, \theta_{d}\right) \in \mathbb{R}^{d}$, the transformation

$$
T_{\theta}:(\mathbb{R} / \mathbb{Z})^{d} \rightarrow(\mathbb{R} / \mathbb{Z})^{d}, \quad T_{\theta}([x])=[x+\theta]
$$

is called the translation of $\mathbb{T}$ by $\theta$.

Let $m$ be the Lebesgue measure on $\mathbb{R}^{d}$. The map

$$
\phi:[0,1]^{d} \rightarrow \mathbb{T}^{d}, \quad \phi(x)=[x]
$$

is surjective and allows us to define a Lebesgue probability measure $\mu$ on the $d$-torus, through the following formula:

$$
\mu(B)=m\left(\phi^{-1}(B)\right), \quad \forall B \subset \mathbb{T}^{d}
$$

such that $\phi^{-1}(B)$ is a measurable subset on $\mathbb{R}^{d}$.

It is not difficult to see that this measure $m$ is invariant under $T_{\theta}$.

Moreover, we say that a vector $\theta=\left(\theta_{1}, \ldots, \theta_{d}\right) \in \mathbb{R}^{d}$ is rationally independent if, for any integer numbers $n_{0}, n_{1}, \ldots, n_{d}$, we have that

$$
n_{0}+n_{1} \theta_{1}+\cdots+n_{d} \theta_{d}=0 \Rightarrow n_{0}=n_{1}=\cdots=n_{d}=0 .
$$

Otherwise, we say that $\theta$ is rationally dependent.

Proposition 2.1.3. The Lebesgue measure $m$ is invariant under every translation map $T_{\theta}$.

Proof. Since $T_{\theta}$ is a continuous map, it follows that $T_{\theta}$ is a measurable function. Define

$$
\mathcal{M}=\left\{E \in \mathcal{B}: m\left(R_{\theta}^{-1}(E)\right)=m(E)\right\}
$$

We claim that $\mathcal{M}=\mathcal{B}$. Indeed, let $I^{d}=[a, b]^{d} \subset \mathbb{T}^{d}$ be a box, then

$$
\begin{aligned}
R_{\theta}^{-1}\left(I^{d}\right)=[a-\theta, b-\theta]^{d} & \Rightarrow m\left(R_{\theta}^{-1}\left(I^{d}\right)\right)=(b-\theta-a+\theta)^{d}=(b-a)^{d}=m\left(I^{d}\right) \\
& \Rightarrow I^{d} \in \mathcal{M} .
\end{aligned}
$$


Hence, $I^{d} \in \mathcal{M}$. On the other hand, let $A=I_{1}^{d} \cup I_{2}^{d} \cup \cdots \cup I_{n}^{d} \in \mathcal{B}$ be a union of disjoint boxes. Then, we have that

$$
\begin{aligned}
m\left(R_{\theta}^{-1}(A)\right) & =m\left(R_{\theta}^{-1}\left(I_{1}^{d} \cup \cdots \cup I_{n}^{d}\right)\right) \\
& =m\left(R_{\theta}^{-1}\left(I_{1}^{d}\right) \cup \cdots \cup R_{\theta}^{-1}\left(I_{n}^{d}\right)\right) \\
& =m\left(R_{\theta}^{-1}\left(I_{1}^{d}\right)\right)+\cdots+m\left(R_{\theta}^{-1}\left(I_{n}^{d}\right)\right) \\
& =m\left(I_{1}^{d}\right)+\cdots+m\left(I_{n}^{d}\right) \\
& =m\left(I_{1}^{d} \cup \cdots \cup I_{n}^{d}\right) . \\
& =m(A) .
\end{aligned}
$$

Then, $A \in \mathcal{M}$. That is, the set $\mathcal{M}$ contains the algebra of finite unions of disjoint boxes. Additionally, $\mathcal{M}$ is a monotone class. Indeed, let $\left\{A_{n}\right\} \subset \mathcal{M}$ be a sequence such that $A_{n} \nearrow A$ then

$$
\begin{aligned}
A_{n} \nearrow A \Rightarrow R_{\theta}^{-1}\left(A_{n}\right) \rightarrow R_{\theta}^{-1}(A) & \Rightarrow m\left(R_{\theta}^{-1}\left(A_{n}\right)\right) \rightarrow m\left(R_{\theta}^{-1}(A)\right) \\
& \Rightarrow m\left(A_{n}\right) \rightarrow m\left(R_{\theta}^{-1}(A)\right) .
\end{aligned}
$$

But, if $A_{n} \nearrow A$ then $m\left(A_{n}\right) \rightarrow m(A)$. Hence, $m\left(R_{\theta}^{-1}(A)\right)=m(A)$.

Analogously, we can show that if $A_{n} \searrow A$, such that $A_{n} \in \mathcal{M}, \forall n \in \mathbb{N}$, then $A \in \mathcal{M}$.

Therefore, $\mathcal{M}$ is a monotone class which contains the Boolean algebra of finite unions of boxes. By the Monotone Class Theorem, $\mathcal{M}$ contains the $\sigma$-algebra generated by this Boolean algebra.

Furthermore, let $A \subset \mathcal{B}$ be a null set. Then, $m(A)=0$ and, for each $\epsilon>0$, there exists $B \in \mathcal{M}$ such that $A \subset B$ and $m(B)<\epsilon$. Hence,

$R_{\theta}^{-1}(A) \subset R_{\theta}^{-1}(B) \Rightarrow m\left(R_{\theta}^{-1}(A)\right) \leq m\left(R_{\theta}^{-1}(B)\right)=m(B)<\epsilon \Rightarrow m\left(R_{\theta}^{-1}(A)\right)<\epsilon$.

Take $\epsilon \rightarrow 0$, then $m\left(R_{\theta}^{-1}(A)\right)=m(A)=0$. Hence, $A \in \mathcal{M}$.

Therefore, $\mathcal{M}=\mathcal{B}$, that is, $m$ is an invariant measure under every translation map $T_{\theta}$.

Proposition 2.1.3 shows that $\left(\mathbb{T}^{d}, m, T_{\theta}\right)$ is a measure preserving dynamical system, for every $\theta \in \mathbb{R}^{d}$. 
Example 2.1.3 (The skew-translations on the 2-torus). Let $f: \mathbb{T}^{2} \rightarrow \mathbb{T}^{2}$ be a map on $\mathbb{T}^{2}$ such that

$$
f\left(\theta_{1}, \theta_{2}\right)=\left(\theta_{1}+\alpha, \theta_{1}+\theta_{2}\right)
$$

where $\alpha$ is an irrational number. Note that $f$ is an invertible function and $f^{-1}\left(\theta_{1}, \theta_{2}\right)=\left(\theta_{1}-\alpha, \theta_{2}-\theta_{1}+\alpha\right)$.

Lemma 2.1.4. Let $M \subset \mathbb{R}^{d}$ be an open subset of $\mathbb{R}^{d}$ and denote by vol the restriction to $M$ of the Lebesgue measure (volume measure) on $\mathbb{R}^{d}$. A $C^{1}$ diffeomorphism $f: M \rightarrow M$ preserves the volume measure vol if, and only if, $|\operatorname{det} D f|=1$ at every point.

Proof. First, suppose that $|\operatorname{det} D f|=1$ at every point. Let $E \subset M$ be a measurable set and $B=f^{-1}(E)$. Then, applying the change of variables formula, we have that

$$
\operatorname{vol}(E)=\int_{B}|\operatorname{det} D f| d x=\int_{B} 1 d x=\operatorname{vol}(B)=\operatorname{vol}\left(f^{-1}(E)\right) .
$$

Hence, $f$ preserves the measure vol.

To prove the second part of the statement, suppose that $|\operatorname{det} D f(x)|>1$ for some point $x \in M$. Since the Jacobian is continuous, there exist a neighborhood $U$ of $x$ and some number $\sigma>1$ such that

$$
|\operatorname{det} D f(y)| \geq \sigma>1, \quad \forall y \in U \text {. }
$$

By the change of variables formula, we have that

$$
\operatorname{vol}(f(U))=\int_{U}|\operatorname{det} D f| d x \geq \int_{U} \sigma d x \geq \sigma \operatorname{vol}(U) .
$$

Denote $E=f(U)$. Since $\operatorname{vol}(U)>0$, the previous inequality implies that $\operatorname{vol}(E)>\operatorname{vol}\left(f^{-1}(E)\right)$. Hence, $f$ does not leave vol invariant. Similarly, one shows that if $|\operatorname{det} D f(x)|<1$ for some point $x \in M$ then $\mathrm{f}$ does not leave the measure vol invariant.

Proposition 2.1.5. The Lebesgue measure $m$ on the torus is $f$-invariant, where $f$ is the skew-translation map defined above.

Proof. Note that the derivative of $f$ at each point is the matrix

$$
\left(\begin{array}{ll}
1 & 0 \\
1 & 1
\end{array}\right)
$$


whose determinant is 1 , hence the result follows from Lemma 2.1.4.

Let $\alpha$ be an irrational number and let $f: \mathbb{T}^{2} \rightarrow \mathbb{T}^{2}$ be the skew-translation map defined above. By the previous proposition, $\left(\mathbb{T}^{2}, m, f\right)$ is a measure preserving dynamical system.

Example 2.1.4 (The Bernoulli shift). Let $(X, \mathcal{C}, \nu)$ be any probability space and define the probability space $\left(\sum, \mathcal{B}, \mu\right)$ such that

(i) $\sum=X^{\mathbb{N}}$ is the set of all sequences $\left(x_{n}\right)_{n \in \mathbb{N}}$ with $x \in X$ for all $n$,

(ii) $\mathcal{B}=\mathcal{C}^{\mathbb{N}}$ is the $\sigma$-algebra generated by the cylinders

$$
\left[m ; A_{m}, \ldots, A_{n}\right]=\left\{\left(x_{i}\right)_{i \in \mathbb{N}}: x_{i} \in A_{i} \text { for all } m \leq i \leq n\right\}
$$

where $m \leq n$ and each $A_{i}$ is an element of $\mathcal{C}$.

(iii) $\mu=\nu^{\mathbb{N}}$ is the product measure, that is,

$$
\mu\left(\left[m ; A_{m}, \ldots, A_{n}\right]\right)=\prod_{i=m}^{n} \nu\left(A_{i}\right)
$$

The map $\sigma: \sum \rightarrow \sum$, where

$$
\sigma\left(\left(x_{n}\right)_{n}\right)=\left(x_{n+1}\right)_{n}
$$

is called the Bernoulli shift. That is, the image of $\left(x_{n}\right)_{n \in \mathbb{N}}=\left(x_{0}, x_{1}, \ldots, x_{n}, \ldots\right)$ is the sequence $\left(x_{n+1}\right)_{n \in \mathbb{N}}=\left(x_{1}, \ldots, x_{n}, \ldots\right)$.

Observe that the image inverse of any cylinder is still a cylinder, that is,

$$
\sigma^{-1}\left(\left[m ; A_{m}, \ldots, A_{n}\right]\right)=\left[m+1 ; A_{m}, \ldots, A_{n}\right] .
$$

Hence,

$$
\begin{aligned}
\mu\left(\sigma^{-1}\left(\left[m ; A_{m}, \ldots, A_{n}\right]\right)\right) & =\mu\left(\left[m+1 ; A_{m}, \ldots, A_{n}\right]\right)=\nu\left(A_{m}\right) \cdots \nu\left(A_{n}\right) \\
& =\mu\left(\left[m ; A_{m}, \ldots, A_{n}\right]\right) .
\end{aligned}
$$

Then, $\mu(E)=\mu\left(\sigma^{-1}(E)\right)$ for every cylinder $E$ in $\mathcal{B}$. Since $\mathcal{B}$ is the $\sigma$-algebra generated by the cylinders, it follows that $\mu(E)=\mu\left(\sigma^{-1} E\right)$ for all $E \in \mathcal{B}$.

Thus, $\mu$ is a $\sigma$-invariant probability measure. 


\section{2}

\section{Ergodic Systems}

Throughout this section, unless otherwise noted, we will suppose that $\mu$ is an invariant probability measure relative to a measurable function $T: M \rightarrow M$.

Definition 2.2.1. Let $(M, \mathcal{B}, \mu, T)$ be a measure preserving dynamical system and let $\varphi: M \rightarrow \mathbb{R}$ be a measurable function.

i) A measurable subset $E \subset X$ is said to be $f$-invariant if $T^{-1} E=E$.

ii) $\varphi: M \rightarrow \mathbb{R}$ is an $T$-invariant function if $\varphi \circ T=\varphi$, for $\mu$-a.e.

Definition 2.2.2. A measure preserving dynamical system $(M, \mathcal{B}, \mu, T)$ is said to be an ergodic system if for all $T$-invariant subsets $E \subset X, \mu(E)=0$ or $\mu(E)=1$.

We say that $\varphi: M \rightarrow \mathbb{R}$ is a $\mu$-a.e. constant function if there exists $c \in \mathbb{R}$ such that $\varphi(x)=c$ for $\mu$-a.e. $x \in M$.

Proposition 2.2.1. Let $\mu$ be an invariant probability measure under a measurable function $T: M \rightarrow M$. Then, $(T, \mu)$ is an ergodic system if and only if every invariant integrable function $\psi: M \rightarrow \mathbb{R}$ is constant $\mu$-a.e.

The following proposition provides other characterizations of ergodicity.

Proposition 2.2.2. Let $T: M \rightarrow M$ be a measurable function and let $\mu$ be a $T$-invariant probability measure on $M$. The following conditions are equivalent:

(a) $(T, \mu)$ is an ergodic system

(b) For any pair of measurable sets $A$ and $B$,

$$
\lim _{n} \frac{1}{n} \sum_{j=0}^{n-1} \mu\left(T^{-j}(A) \cap B\right)=\mu(A) \mu(B)
$$

(c) For every map $\phi \in L^{p}(\mu)$ and every map $\psi \in L^{q}(\mu)$, with $\frac{1}{p}+\frac{1}{q}=1$, we have

$$
\lim _{n} \frac{1}{n} \sum_{j=0}^{n-1} \int(\varphi \circ T)^{j} \psi d \mu=\int \varphi d \mu \int \psi d \mu
$$

To prove that the previous examples are also ergodic systems, we begin with a brief exposition about Fourier series. 
Let $(\Omega, M, \mu)$ be a measure space and let $p \in \mathbb{R}$ with $1<p<\infty$, we set

$$
L^{p}(\Omega)=\left\{f: \Omega \rightarrow \mathbb{R}: f \text { is measurable and }|f|^{p} \in L^{1}(\Omega)\right\}
$$

with

$$
\|f\|_{L^{p}}=\|f\|_{p}=\left[\int_{\Omega}|f(x)|^{p} d \mu\right]^{1 / p}
$$

In particular, $L^{2}(\mathbb{T})$ is the space of all square integrable functions on $\mathbb{T}$ and $L^{2}(\mathbb{T})$ is a Hilbert space endowed with the inner product

$$
\langle f, g\rangle=\int_{\mathbb{T}} f \bar{g}
$$

Let $e: \mathbb{T} \rightarrow \mathbb{C}, e(x):=e^{2 \pi i x}$. It is known that the maps $\left\{e_{n}\right\}_{n \in \mathbb{Z}}$, where

$$
e_{n}(x):=e(n x)=e^{2 \pi i n x}
$$

form an orthonormal basis in $L^{2}(\mathbb{T})$.

Given a function $f \in L^{2}(\mathbb{T})$, its $n$-th Fourier coefficient is

$$
\hat{f}(n):=\int_{\mathbb{T}} f(t) \overline{e_{n}(t)} d t=\int_{\mathbb{T}} f(t) e(-n t) d t .
$$

Thus, any $f \in L^{2}(T)$ may be expanded into the Fourier series

$$
f(x)=\sum_{n \in \mathbb{Z}} \hat{f}(n) e_{n}(x)
$$

where the convergence (or equality) above is understood in the $L^{2}$ sense.

The interested reader may find all the details regarding the Fourier analysis concepts used in this dissertation in [8] and [9].

Proposition 2.2.3. The one-dimensional torus translation $\left(\mathbb{T}, m, T_{\alpha}\right)$ is an ergodic system if and only if $\alpha$ is an irrational number.

Proof. First, we will prove that if $\alpha \in \mathbb{Q}$, then $\left(\mathbb{T}, m, T_{\alpha}\right)$ is not ergodic.

Let $\alpha=\frac{p}{q} \in \mathbb{Q}$ and let $I \subset \mathbb{T}$ be a segment with length less than $\frac{1}{q}$. Then, $T_{\alpha}^{q}(x)=x, \forall x \in \mathbb{T}$ and the set

$$
A=I \cup T_{\alpha}(I) \cup \cdots \cup T_{\alpha}^{q-1}(I)
$$


is invariant. That is because

$$
\begin{aligned}
T_{\alpha}(A) & =T_{\alpha}\left(I \cup T_{\alpha}(I) \cup \cdots \cup T_{\alpha}^{q-1}(I)\right) \\
& =T_{\alpha}(I) \cup T_{\alpha}^{2}(I) \cup \cdots \cup T_{\alpha}^{q-1}(I) \cup T_{\alpha}^{q}(I) \\
& =I \cup T_{\alpha}(I) \cup T_{\alpha}^{2}(I) \cup \cdots \cup T_{\alpha}^{q-1}(I) \\
& =A
\end{aligned}
$$

and, since $T_{\alpha}$ is a bijective function, we have that $T_{\alpha}^{-1}(A)=A$.

On the other hand,

$$
\begin{aligned}
m(A) & =m\left(I \cup T_{\alpha}(I) \cup \cdots \cup T_{\alpha}^{q-1}(I)\right) \\
& =m(I)+m\left(T_{\alpha}(I)\right)+\cdots+m\left(T_{\alpha}^{q-1}(I)\right) \\
& =\underbrace{m(I)+\cdots+m(I)}_{q \text { times }} \\
& =q \cdot m(I) \\
& <1 .
\end{aligned}
$$

Moreover, if $I \subset A$ then $0<m(I) \leq m(A)$. Thus, $0<m(A)<1$ and, since $A$ is an invariant set, we conclude that $T_{\alpha}$ is not ergodic.

Reversely, suppose that $\alpha$ is an irrational number and let $\varphi \in L^{2}(\mathbb{T})$ be an invariant function. We consider the Fourier expansion of $\varphi$. That is, if we denote by $\hat{\varphi}(k)$ the $k$-Fourier coefficient of $\varphi$, then

$$
\varphi(x)=\sum_{k \in \mathbb{Z}} \widehat{\varphi}(k) e^{2 \pi i k x} \quad \text { in } L^{2}(\mathbb{T})
$$

Moreover, if $\widehat{\varphi \circ T_{\alpha}}(k)$ denote the $k$-Fourier coefficient of $\varphi \circ T_{\alpha}$ and applying the change of variables formula, we have that

$$
\widehat{\varphi \circ T_{\alpha}}(k)=\int_{\mathbb{T}} \varphi(x+\alpha) e^{-2 \pi i k x} d x=\int_{\mathbb{T}} \varphi(y) e^{-2 \pi i k y} e^{2 \pi i k \alpha}=\widehat{\varphi}(k) e^{e \pi i k \alpha} .
$$

Hence,

$$
\left(\varphi \circ T_{\alpha}\right)(x)=\sum_{k \in \mathbb{Z}} \widehat{\varphi \circ T_{\alpha}}(k) e^{2 \pi i k x}=\sum_{k \in \mathbb{Z}} \widehat{\varphi}(k) e^{e \pi i k \alpha} e^{2 \pi i k x} .
$$

Since $\varphi$ is a $T_{\alpha}$-invariant function, $\varphi=\varphi \circ T_{\alpha}$. On the other hand, the coefficients of Fourier series are unique, then

$$
\widehat{\varphi}(k)=\widehat{\varphi}(k) e^{e \pi i k \alpha}, \quad \text { for all } k \in \mathbb{Z} \text {. }
$$


But $e^{2 \pi i k \theta} \neq 1$, for all $k \neq 0$ since $\alpha \notin \mathbb{Q}$. Then, $\widehat{\varphi}(k)=0, \forall k \neq 0$. That is, $\varphi(z)=a_{0}$ for $m$-a.e. $z \in \mathbb{T}$.

In particular, the indicator function $\varphi=\mathbb{1}_{A}$ of any invariant subset $A \subset \mathbb{T}$ is an $m$-a.e. constant function. Then, $m(A)=0$ or $m(A)=1$, that is, $\left(T_{\alpha}, m\right)$ is ergodic.

This proposition can be extended to the translation map on the $d$-torus, for every $d \geq 1$.

Proposition 2.2.4. If $\theta=\left(\theta_{1}, \ldots, \theta_{d}\right)$ is rationally independent then the translation map $T_{\theta}: \mathbb{T}^{d} \rightarrow \mathbb{T}^{d}$ is ergodic.

Proof. Suppose that $\theta=\left(\theta_{1}, \ldots, \theta_{d}\right)$ is a rationally independent vector and let $\varphi: \mathbb{T}^{d} \rightarrow \mathbb{C} \in L^{2}\left(\mathbb{T}^{d}\right)$ be an invariant map, that is, $\varphi \circ T_{\theta}=\varphi$ for $m$-a.e.

We consider the Fourier expansion of $\varphi$. That is, if we denote by $\widehat{\varphi}(k)$ the $k$-Fourier coefficient of $\varphi$, then

$$
\varphi(x)=\sum_{k \in \mathbb{Z}^{d}} \widehat{\varphi}(k) e^{2 \pi i k \cdot x}, \quad \text { in } L^{2}\left(\mathbb{T}^{d}\right)
$$

where $x=\left(x_{1}, \ldots, x_{d}\right) \in \mathbb{T}^{d}, k=\left(k_{1}, \ldots, k_{d}\right) \in \mathbb{Z}^{d}$ and $k \cdot x=k_{1} x_{1}+\cdots+k_{d} x_{d}$.

Analogously by Proposition 2.2.3, we may prove that

$$
\widehat{\varphi \circ T_{\theta}}(k)=\widehat{\varphi(k)} e^{2 \pi i k \cdot \theta} .
$$

Hence,

$$
\left(\varphi \circ T_{\theta}\right)(x)=\sum_{k \in \mathbb{Z}^{d}} \widehat{\varphi \circ T_{\theta}}(k) e^{2 \pi i k \cdot x}=\sum_{k \in \mathbb{Z}^{d}} \widehat{\varphi(k)} e^{2 \pi i k \cdot \theta} e^{2 \pi i k \cdot x} .
$$

Since $\varphi$ is an invariant map, the above equations are equal. Moreover, since the coefficients of Fourier series are unique, we have

$$
\widehat{\varphi(k)} e^{2 \pi i k \cdot \theta}=\widehat{\varphi(k)}, \quad \text { for all } k \in \mathbb{Z}^{d}
$$

But $e^{2 \pi i k \cdot \theta} \neq 1$, for all $k \neq 0$ since $\theta=\left(\theta_{1}, \ldots, \theta_{d}\right)$ is rationally independent. Then, $\widehat{\varphi(k)}=0, \forall k \neq 0$. That is, $\varphi(z)=a_{0}$ for $m$-a.e. $z \in \mathbb{T}^{d}$.

Hence, the indicator function $\varphi=\mathbb{1}_{A}$ of any invariant subset $A \subset \mathbb{T}^{d}$ is an $m$-a.e. constant function. Then, $\left(T_{\theta}, m\right)$ is ergodic.

Proposition 2.2.5. Let $f$ be the skew-translation map defined in Example 2.1.3. Then $f$ is ergodic (relative to the Lebesgue measure $m$ ). 
Proof. Let $\varphi: \mathbb{T}^{2} \rightarrow \mathbb{R}$ be a function in $L^{2}\left(\mathbb{T}^{2}\right)$. If we denote by $\widehat{\varphi}(k)$ the $k$-Fourier coefficient of $\varphi$, then the Fourier expansion of $\varphi$ is given by

$$
\varphi(\theta)=\sum_{n \in \mathbb{Z}^{2}} \widehat{\varphi}(n) e^{2 \pi i n \cdot \theta}, \quad \text { in } L^{2}\left(\mathbb{T}^{2}\right)
$$

where $\theta=\left(\theta_{1}, \theta_{2}\right) \in \mathbb{T}^{2}, n=\left(n_{1}, n_{2}\right) \in \mathbb{Z}^{2}$ and $n \cdot \theta=n_{1} \theta_{1}+n_{2} \theta_{2}$.

Moreover, by the Plancherel theorem

$$
\sum_{n \in \mathbb{Z}^{2}}|\widehat{\varphi}(n)|^{2}=\int\left|\varphi\left(\theta_{1}, \theta_{2}\right)\right|^{2} d \theta_{1} d \theta_{2}<\infty
$$

Furthermore,

$$
\begin{aligned}
\varphi\left(f\left(\theta_{1}, \theta_{2}\right)\right) & =\sum_{n \in \mathbb{Z}^{2}} \widehat{\varphi}(n) e^{2 \pi i\left(n_{1}\left(\theta_{1}+\alpha\right)+n_{2}\left(\theta_{2}+\theta_{1}\right)\right)} \\
& =\sum_{n \in \mathbb{Z}^{2}} \widehat{\varphi}(n) e^{2 \pi i n_{1} \alpha} e^{2 \pi i\left(n_{1} \theta_{1}+n_{2}\left(\theta_{2}+\theta_{1}\right)\right)} \\
& =\sum_{n \in \mathbb{Z}^{2}} \widehat{\varphi}(n) e^{2 \pi i n_{1} \alpha} e^{2 \pi i\left(\left(n_{1}+n_{2}\right) \theta_{1}+n_{2} \theta_{2}\right)}
\end{aligned}
$$

Suppose that $\varphi$ is $f$-invariant, that is, $\varphi \circ f=\varphi$, for $m$-a.e. Hence, by the uniqueness of the Fourier coefficients, we have that

$$
\widehat{\varphi}(n) e^{2 \pi i n_{1} \alpha}=\widehat{\varphi}(L(n)), \quad \text { for all } n \in \mathbb{Z}^{2}
$$

where $L(n)=\left(n_{1}+n_{2}, n_{2}\right)$.

Then, $|\widehat{\varphi}(n)|=\left|\widehat{\varphi}(n) e^{2 \pi i n_{1} \alpha}\right|=|\widehat{\varphi}(L(n))|$. By (2.2), we conclude that $\widehat{\varphi}(n)=0$ for all $n \in \mathbb{Z}^{2}$ such that the orbit $L^{j}(n), j \in \mathbb{Z}$ is infinite. In particular, $\widehat{\varphi}(n)=0$ where $n=\left(n_{1}, n_{2}\right)$ with $n_{2} \neq 0$. On the other hand, if $n=\left(n_{1}, 0\right)$ then $L(n)=n$. Hence, the relation (2.3) becomes

$$
\widehat{\varphi}(n) e^{2 \pi i n_{1} \alpha}=\widehat{\varphi}(n)
$$

Since $\alpha$ is an irrational number, $\widehat{\varphi}(n)=0$ when $n=\left(n_{1}, 0\right)$ with $n_{1} \neq 0$. Hence, $\varphi$ is $m$-a.e. constant function and we conclude that $f$ is ergodic.

The next lemma is a technical step towards proving the ergodicity of the Bernoulli shift.

Lemma 2.2.6. If $B$ and $C$ are finite unions of pairwise disjoint cylinders, then for every $j \in \mathbb{N}$ large enough,

$$
\mu\left(B \cap \sigma^{-j}(C)\right)=\mu(B) \mu\left(\sigma^{-j}(C)\right)=\mu(B) \mu(C) .
$$


Proof. First, suppose that $B$ and $C$ are cylinders, that is,

$$
B=\left[k ; B_{k}, \ldots, B_{l}\right] \text { and } C=\left[m ; C_{m}, \ldots, C_{n}\right]
$$

It is easy to check that

$$
\sigma^{-j}(C)=\left[m+j ; C_{m}, \ldots, C_{n}\right]
$$

Take $j$ large enough such that $m+j>l$ for some $l \in \mathbb{N}$. Then,

$$
\begin{aligned}
B \cap \sigma^{-j}(C) & =\left\{\left(x_{n}\right)_{n}: x_{k} \in B_{k}, \ldots, x_{l} \in B_{l}, x_{m+j} \in C_{m}, \ldots, x_{n+j} \in C_{n}\right\} \\
& =[k ; B_{k}, \ldots, B_{l}, \underbrace{X, \ldots, X}_{m+j-l-1 \text { times }} C_{m}, \ldots, C_{n}] .
\end{aligned}
$$

Hence,

$$
\begin{aligned}
\mu\left(B \cap \sigma^{-j}(C)\right) & =\mu\left([k ; B_{k}, \ldots, B_{l}, \underbrace{X, \ldots, X}_{m+j-l-1 \text { times }}, C_{m}, \ldots, C_{n}]\right) \\
& =\prod_{i=k}^{l} \nu\left(B_{i}\right) \prod_{i=1}^{m+j-l-1} \nu(X) \prod_{i=m}^{n} \nu\left(C_{i}\right) \\
& =\prod_{i=k}^{l} \nu\left(B_{i}\right) \prod_{i=m}^{n} \nu\left(C_{i}\right) \\
& =\mu(B) \cdot \mu(C)
\end{aligned}
$$

since $\nu(X)=1$.

This completes the proof when $A$ and $B$ are cylinders. The general case follows from the fact that $\mu$ is finitely additive.

Proposition 2.2.7. Let $\sigma$ be the Bernoulli shift defined in the Example 2.1.4. Then, $\sigma$ is ergodic.

Proof. Let $\mathcal{B}_{0}$ be the algebra of all unions of pairwise disjoint cylinders and let $A \in \mathcal{B}_{0}$ be a $\sigma$-invariant measurable subset of $\sum$.

Applying the Lemma 2.2.6 with $B=C=A$, we obtain

$$
\mu\left(A \cap \sigma^{-j}(A)\right)=\mu(A)^{2}
$$

for $j$ large enough.

Since $A$ is invariant, $\mu\left(A \cap \sigma^{-j}(A)\right)=\mu(A)$. Hence, $\mu(A)=\mu(A)^{2}$, and, consequently, $\mu(A)=0$ or $\mu(A)=1$. 
Now, suppose that $A \subset \sum$ is any $\sigma$-invariant measurable subset. For every $\epsilon>0$, there exists $B \in \mathcal{B}_{0}$ such that $\mu(A \triangle B)<\epsilon$, recalling that $A \triangle B:=(A \backslash B) \cup(B \backslash A)$. Fix $j$ such that

$$
\mu\left(B \cap \sigma^{-j}(B)\right)=\mu(B) \mu\left(\sigma^{-j}(B)\right)=\mu(B)^{2} .
$$

Observe that

$$
(A \triangle B) \cup\left(\sigma^{-j}(A) \triangle \sigma^{-j}(B)\right)=(A \triangle B) \cup \sigma^{-j}(A \triangle B)
$$

and

$$
\left(A \cap \sigma^{-j}(A)\right) \triangle\left(B \cap \sigma^{-j}(B) \subset(A \triangle B) \cup\left(\sigma^{-j}(A) \triangle \sigma^{-j}(B)\right)\right.
$$

Hence,

$$
\begin{aligned}
\left|\mu(\underbrace{A \cap \sigma^{-j}(A)}_{X})-\mu(\underbrace{B \cap \sigma^{-j}(B)}_{Y})\right| & =|\mu(X \backslash Y)-\mu(Y \backslash X)| \\
& \leq|\mu(X \backslash Y)|+|\mu(Y \backslash X)| \\
& =\left|\mu\left(A \cap \sigma^{-j}(A)\right) \triangle\left(B \cap \sigma^{-j}(B)\right)\right| \\
& \leq \mu\left((A \triangle B) \cup\left(\sigma^{-j}(A) \triangle \sigma^{-j}(B)\right)\right) \\
& \leq 2 \mu(A \triangle B)<2 \epsilon
\end{aligned}
$$

since $\mu$ is a $\sigma$-invariant measure.

Combining all of the above, we conclude that

$$
\left|\mu(A)-\mu(A)^{2}\right|<4 \epsilon \text {. }
$$

By the arbitrariness of $\epsilon$, it follows that $\mu(A)=\mu(A)^{2}$, that is, $\mu(A)=0$ or $\mu(A)=1$.

\section{3}

\section{The Pointwise and Subadditive Ergodic Theorems}

In this section, we present the proof of one of the most important results in ergodic theory, Birkhoff's pointwise ergodic theorem. We follow the argument of Y. Katznelson and B. Weiss [10] since it is more related to the spirit of this dissertation. However, other proofs are available, see for instance the book of M.Viana and K. Oliveira [4]. 
We also formulate Kingman's subadditive ergodic theorem, whose proof in [10] is similar to that of Birkhoff's ergodic theorem.

We start with the pointwise ergodic theorem.

Theorem 2.3.1 (Birkhoff for measure preserving transformations). Let $(X, \mathcal{B}, \mu, T)$ be a measure preserving dynamical system and let $f: X \rightarrow \mathbb{R}$ be an integrable function. Then, the limit

$$
\lim _{n \rightarrow \infty} \frac{f(x)+f(T x)+\cdots+f\left(T^{n-1} x\right)}{n}=: f^{*}(x)
$$

exists for almost every $x \in X$. Moreover, $f^{*}$ is a T-invariant function and $\int_{X} f^{*} d \mu=\int_{X} f d \mu$.

Proof. (Following [10]) Suppose that $f$ is a non-negative function and define

$$
\sigma_{n}(x)=\frac{f(x)+f(T x)+\cdots+f\left(T^{n-1} x\right)}{n} .
$$

We need to show that the limit $\lim _{n \rightarrow \infty} \sigma_{n}(x)$ exists for $\mu$-a.e. $x \in X$.

Define

$$
\bar{f}(x):=\limsup _{n \rightarrow \infty} \sigma_{n}(x) \text { and } \quad \underline{f}(x):=\liminf _{n \rightarrow \infty} \sigma_{n}(x) .
$$

Hence, $\underline{f}(x) \leq \bar{f}(x)$ for every $x \in X$.

Observe that

$$
\begin{aligned}
\sigma_{n+1}(x) & =\frac{1}{n+1}\left(f(x)+f(T x)+\cdots+f\left(T^{n} x\right)\right) \\
& =\frac{1}{n+1} f(x)+\frac{n}{n+1} \sigma_{n}(T x)
\end{aligned}
$$

Since, $\lim _{n \rightarrow \infty} \frac{1}{n+1} f(x)=0$ and $\lim _{n \rightarrow \infty} \frac{n}{n+1}=1$, we have

$$
\bar{f}(x)=\limsup _{n \rightarrow \infty} \sigma_{n+1}(x)=\limsup _{n \rightarrow \infty} \sigma_{n}(T x)=\bar{f}(T x) .
$$

Similarly, $\underline{f}(x)=\underline{f}(T x)$, that is, $\bar{f}$ and $\underline{f}$ are $T$-invariant functions.

We want to prove that $\bar{f}(x)=\underline{f}(x)$ for $\mu$-a.e. $x \in X$. First, we will show that

$$
\int_{X} \bar{f} d \mu \leq \int_{X} f d \mu \leq \int_{X} \underline{f} d \mu .
$$

We only prove first inequality in (2.5), as the proof of the second inequality is analogous. We claim that

$$
\int_{X} \bar{f} d \mu \leq \int_{X} f d \mu+O(\epsilon)
$$


for every $\epsilon>0$. Indeed, for this, we will prove that

$$
\frac{1}{L} \sum_{j=0}^{L-1} \bar{f}\left(T^{j} x\right) \leq \frac{1}{L} \sum_{j=0}^{L-1} f\left(T^{j} x\right)+O(\epsilon) .
$$

We can suppose that there exists $M \in \mathbb{R}$ such that $\bar{f}(x) \leq M$ for every $x \in X$. Otherwise, define $\bar{f}_{M}(x)=\min \{f(x), M\}$. Then, $\bar{f}_{M} \nearrow f$ and the function $\bar{f}_{M}$ is $T$-invariant since $\bar{f}$ is $T$-invariant. Hence, if the function $\bar{f}_{M}$ satisfies the inequality (2.6) then the same inequality is true for $\bar{f}$ by the monotone convergence theorem.

By the definition of $\bar{f}$, for every $x \in X$, there exists $n \in \mathbb{N}$ such that

$$
\bar{f}(x)-\epsilon \leq \frac{1}{n} \sum_{j=0}^{n-1} f\left(T^{j} x\right)
$$

that is, $n \bar{f}(x) \leq f(x)+\cdots+f\left(T^{n-1} x\right)+n \epsilon$.

Since $\bar{f}$ is a $T$-invariant function, we have that

$$
\bar{f}(x)=\bar{f}(T x)=\cdots=\bar{f}\left(T^{n-1} x\right) \Rightarrow n \bar{f}(x)=\bar{f}(x)+\bar{f}(T x)+\cdots+\bar{f}\left(T^{n-1} x\right) .
$$

Then, for each $x \in X$, there exists $n \in \mathbb{N}$ such that

$$
\sum_{j=0}^{n-1} \bar{f}\left(T^{j} x\right) \leq \sum_{j=0}^{n-1} f\left(T^{j} x\right)+n \epsilon .
$$

Let $n(x)$ be the first $n$ such that the inequality (2.7) is satisfied. Then,

$$
\sum_{j=0}^{n(x)-1} \bar{f}\left(T^{j} x\right) \leq \sum_{j=0}^{n(x)-1} f\left(T^{j} x\right)+n(x) \epsilon
$$

For each $N \in \mathbb{N}$, define $\mathcal{A}_{N}=\{x \in X: n(x) \leq N\}$. Hence, $\mathcal{A}_{N} \nearrow X$ since for each $x \in X$, there exists $n(x) \in \mathbb{N}$. Consequently, $\mu\left(\mathcal{A}_{n}\right) \rightarrow \mu(X)=1$. Thus, there exists $N \in \mathbb{N}$ such that $\mu\left(X \backslash \mathcal{A}_{N}\right)<\epsilon / M$.

Fix $N \in \mathbb{N}$ such that $\mu\left(X \backslash \mathcal{A}_{N}\right)<\epsilon / M$ and put $\mathcal{A}:=\mathcal{A}_{N}$. Hence, if $x \in \mathcal{A}$ then $1 \leq n(x) \leq N$ and

$$
\sum_{j=0}^{n(x)-1} \bar{f}\left(T^{j} x\right) \leq \sum_{j=0}^{n(x)-1} f\left(T^{j} x\right)+n(x) \epsilon .
$$

Fix $x \in X$. We define a sequence of indices $\left\{n_{j}\right\}$ and points $\left\{x_{j}\right\}$ by the 
following rule:

$$
x_{1}=x, \quad \text { and } \quad n_{1}= \begin{cases}n\left(x_{1}\right), & \text { if } x_{1} \in \mathcal{A} \\ 1, & \text { if } x_{1} \notin \mathcal{A}\end{cases}
$$

And, for $j \geq 1$, define

$$
x_{j+1}=T^{n_{j}} x_{j}, \quad \text { and } \quad n_{j+1}= \begin{cases}n\left(x_{j+1}\right), & \text { if } x_{j+1} \in \mathcal{A} \\ 1, & \text { if } x_{j+1} \notin \mathcal{A}\end{cases}
$$

that is, $x_{j+1}=T^{n_{1}+\cdots+n_{j}} x$.

Fix $L \in \mathbb{N}$ such that $L \geq N \cdot \frac{M}{\epsilon}>N$. Since $1 \leq n_{j} \leq N$, it follows that the sequence $a_{n}=\sum_{j=1}^{n} n_{j}$ is such that $a_{n} \nearrow \infty$. Hence, there exists $p \in \mathbb{N}$ such that

$$
n_{1}+\cdots+n_{p} \leq L \leq n_{1}+\cdots+n_{p+1},
$$

that is, there exists $K \in\left\{0, \ldots, n_{p+1}\right\}$ such that $L=n_{1}+\cdots+n_{p}+K$. Observe that

$$
\begin{gathered}
\sum_{j=0}^{L-1} \bar{f}\left(T^{j} x\right)=\sum_{j=0}^{n_{1}-1} \bar{f}\left(T^{j} x\right)+\sum_{j=n_{1}}^{n_{1}+n_{2}-1} \bar{f}\left(T^{j} x\right)+\cdots+\sum_{j=n_{1}+\cdots+n_{p-1}}^{n_{1}+\cdots+n_{p}-1} \bar{f}\left(T^{j} x\right)+ \\
+\sum_{j=n_{1}+\cdots+n_{p}}^{L-1} \bar{f}\left(T^{j} x\right) .
\end{gathered}
$$

Denote, respectively, by $S_{1}(x), S_{2}(x), \ldots, S_{p}(x), S_{p+1}(x)$ the $p+1$ sums in the previous equality. From (2.7), we get

$$
S_{1}(x)=\sum_{j=0}^{n\left(x_{1}\right)-1} \bar{f}\left(T^{j} x_{1}\right) \leq \sum_{j=0}^{n\left(x_{1}\right)-1} f\left(T^{j} x_{1}\right)+n\left(x_{1}\right) \epsilon, \quad \text { if } x_{1}=x \in \mathcal{A}
$$

but, if $x_{1}=x \notin \mathcal{A}$ then $n_{1}=1$ and $S_{1}(x)=\bar{f}\left(x_{1}\right) \leq M$. On the other hand,

$$
\left(\sum_{j=0}^{n_{1}-1} f\left(T^{j} x\right)+n_{1} \epsilon\right) \mathbb{1}_{\mathcal{A}}(x) \leq \sum_{j=0}^{n_{1}-1} f\left(T^{j} x\right)+n_{1} \epsilon
$$

since we assume that $f \geq 0$. Hence,

$$
S_{1}(x) \leq\left(\sum_{j=0}^{n_{1}-1} f\left(T^{j} x\right)+n_{1} \epsilon\right)+M \cdot \mathbb{1}_{X \backslash \mathcal{A}}\left(x_{1}\right) .
$$

For the second sum, take $j=n_{1}+l$ with $l=0, \ldots, n_{2}-1$. Then, we can 
rewrite the sum as:

$$
S_{2}(x)=\sum_{j=n_{1}}^{n_{1}+n_{2}-1} \bar{f}\left(T^{j} x\right)=\sum_{l=0}^{n_{2}-1} \bar{f}\left(T^{l} T^{n_{1}} x\right)=\sum_{l=0}^{n_{2}-1} \bar{f}\left(T^{l} x_{2}\right)
$$

and we can use a similar argument as the first sum $S_{1}(x)$.

Hence,

$$
S_{2}(x) \leq\left(\sum_{l=0}^{n_{2}-1} f\left(T^{l} x_{2}\right)+n_{2} \epsilon\right)+M \cdot \mathbb{1}_{X \backslash \mathcal{A}}\left(x_{2}\right)
$$

Inductively,

$$
S_{p}(x) \leq\left(\sum_{l=0}^{n_{p}-1} f\left(T^{l} x_{p}\right)+n_{p} \epsilon\right)+M \cdot \mathbb{1}_{X \backslash \mathcal{A}}\left(x_{p}\right)
$$

and, since $\bar{f}(x) \leq M$ for every $x \in X$, we have that

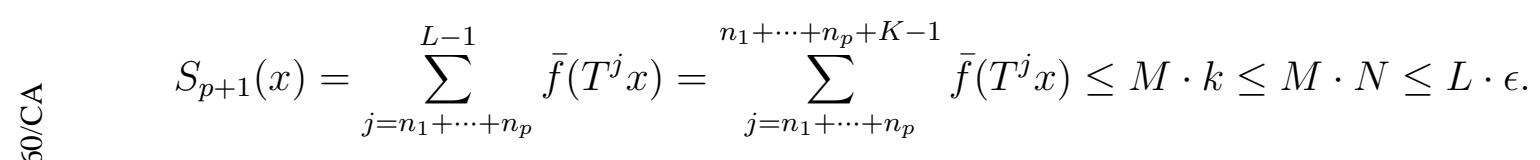

Observe that

$$
\begin{aligned}
\mathbb{1}_{X \backslash \mathcal{A}}\left(x_{1}\right)+\cdots+\mathbb{1}_{X \backslash \mathcal{A}}\left(x_{p}\right) & =\mathbb{1}_{X \backslash \mathcal{A}}\left(x_{1}\right)+\cdots+\mathbb{1}_{X \backslash \mathcal{A}}\left(T^{n_{1}+\cdots+n_{p}} x_{1}\right) \\
& \leq \sum_{j=0}^{L-1} \mathbb{1}_{X \backslash \mathcal{A}}\left(T^{j} x\right)
\end{aligned}
$$

Hence,

$$
\begin{aligned}
\sum_{j=0}^{L-1} \bar{f}\left(T^{j} x\right) & =\left(S_{1}(x)+\cdots+S_{p}(x)\right)+S_{p+1}(x) \\
& \leq \sum_{j=0}^{n_{1}+\cdots+n_{p}-1} f\left(T^{l} x\right)+\left(n_{1}+\cdots+n_{p}\right) \epsilon+M \cdot \sum_{i=1}^{p} \mathbb{1}_{X \backslash \mathcal{A}}\left(x_{i}\right)+L \epsilon \\
& \leq \sum_{j=0}^{L-1} f\left(T^{j} x\right)+L \cdot \epsilon+M \cdot\left(\mathbb{1}_{X \backslash \mathcal{A}}\left(x_{1}\right)+\cdots+\mathbb{1}_{X \backslash \mathcal{A}}\left(x_{p}\right)\right)+L \epsilon \\
& \leq \sum_{j=0}^{L-1} f\left(T^{j} x\right)+L(2 \epsilon)+M \cdot \sum_{j=0}^{L-1} \mathbb{1}_{X \backslash \mathcal{A}}\left(T^{j} x\right) .
\end{aligned}
$$

Then,

$$
\frac{1}{L} \sum_{j=0}^{L-1} \bar{f}\left(T^{j} x\right) \leq \frac{1}{L} \sum_{j=0}^{L-1} f\left(T^{j} x\right)+M \cdot\left(\frac{1}{L} \sum_{j=0}^{L-1} \mathbb{1}_{X \backslash \mathcal{A}}\left(T^{j} x\right)\right)+2 \epsilon
$$


for every $x \in X$.

Since the transformation $T$ preserves the measure $\mu$, we have that for every $j \in \mathbb{N}, \int_{X} \bar{f}\left(T^{j} x\right) d \mu(x)=\int_{X} \bar{f} d \mu, \int_{X} f\left(T^{j} x\right) d \mu(x)=\int_{X} f d \mu$ and $\int_{X} \mathbb{1}_{X \backslash \mathcal{A}}\left(T^{j} x\right) d \mu(x)=\int_{X} \mathbb{1}_{X \backslash \mathcal{A}} d \mu$

Integrating the inequality (2.8) and using the fact that $L$ is independent of $x$, we have that

$$
\begin{aligned}
\int_{X} \bar{f} d \mu & =\frac{1}{L} \sum_{j=0}^{L-1} \int_{X} \bar{f}\left(T^{j} x\right) \\
& \leq \frac{1}{L} \sum_{j=0}^{L-1} \int_{X} f\left(T^{j} x\right)+M \cdot\left(\frac{1}{L} \sum_{j=0}^{L-1} \int_{X} \mathbb{1}_{X \backslash \mathcal{A}}\left(T^{j} x\right)\right)+2 \epsilon \\
& \leq \int_{X} f d \mu+M \int_{X} \mathbb{1}_{X \backslash \mathcal{A}} d \mu+2 \epsilon \\
& =\int_{X} f d \mu+2 \epsilon+M \mu\left(\mathcal{A}^{c}\right) \\
& \leq \int_{X} f d \mu+3 \epsilon .
\end{aligned}
$$

Letting $\epsilon \rightarrow 0$, we conclude that

$$
\int_{X} \bar{f} d \mu \leq \int_{X} f d \mu
$$

Similarly, we can prove that $\int_{X} f d \mu \leq \int_{X} \bar{f} d \mu$. Hence,

$$
\int_{X} \bar{f} d \mu \leq \int_{X} f d \mu \leq \int_{X} f d \mu .
$$

Moreover, we know that $\int_{X} \underline{f} d \mu \leq \int_{X} \bar{f} d \mu$. Hence, $\int_{X} \bar{f} d \mu=\int_{X} \underline{f} d \mu$ and, consequently,

$$
\int_{X}(\bar{f}-\underline{f}) d \mu=0 \Rightarrow \bar{f}=\underline{f}, \quad \mu \text {-a.e. }
$$

that is, $\liminf _{n \rightarrow \infty} \sigma_{n}(x)=\limsup _{n \rightarrow \infty} \sigma_{n}(x)$.

Then, the limit $\lim _{n \rightarrow \infty} \sigma_{n}(x)$ exists and $f^{*}=\bar{f}$. Moreover,

$$
\int_{X} f^{*} d \mu=\int_{X} \bar{f} d \mu=\int_{X} f d \mu .
$$

If $f$ is not a non-negative function, then we can write $f=f^{+}-f^{-}$, with $f^{+}, f^{-} \geq 0$ and apply the result above to $f^{+}$and $f^{-}$.

In particular for ergodic system, we obtain the following. 
Corollary 2.3.1 (Birkhoff's Ergodic Theorem for ergodic transformations). Let $(X, \mathcal{B}, \mu, T)$ be an ergodic system. Then, for every integrable function $f: X \rightarrow \mathbb{R}$, we have that

$$
\lim _{n \rightarrow \infty} \frac{f(x)+f(T x)+\cdots+f\left(T^{n-1} x\right)}{n}=\int_{X} f(x) d \mu(x)
$$

for $\mu$-a.e. $x \in X$.

Proof. By the Birkhoff Ergodic Theorem for measure preserving transformations, the limit

$$
\lim _{n \rightarrow \infty} \frac{f(x)+f(T x)+\cdots+f\left(T^{n-1} x\right)}{n}=f^{*}(x)
$$

exists for $\mu$-a.e. $x \in X$. Moreover, $f^{*}$ is a $T$-invariant function and

$$
\int_{X} f^{*} d \mu=\int_{X} f d \mu
$$

Since $T$ is ergodic, there exists $c \in \mathbb{R}$ such that $f^{*}(x)=c$ for $\mu$-a.e. $x \in X$. Hence,

$$
\int_{X} f^{*} d \mu=c \cdot \mu(X)=c
$$

Thus, from (2.9) and (2.10) we get $\int_{X} f d \mu=c$. Then, for almost every $x \in X$

$$
\lim _{n \rightarrow \infty} \frac{1}{n} \sum_{k=0}^{n-1} f\left(T^{k}(x)\right)=c=\int_{X} f d \mu
$$

which is the conclusion we were looking for.

The quantity in (2.4),

$$
S_{n} f(x):=\sum_{j=0}^{n-1} f\left(T^{j} x\right)
$$

is called the Birkhoff sums of the observable ${ }^{1} f$ with respect to the map $T$.

In general, a subset of total measure which satisfies the convergence (2.4) in the Theorem 2.3.1 depends of the observable $f$ considered.

However, in some cases it is possible to choose this subset independently of this function. A useful example of this situation is the following:

Theorem 2.3.2. Let $M$ be a compact metric space and $T: M \rightarrow M$ be a continuous map. Then, there exists a measurable subset $G$ on $M$ with total 
measure $\mu(G)=1$ such that

$$
\lim _{n \rightarrow \infty} \frac{1}{n} \sum_{k=0}^{n-1} f\left(T^{k}(x)\right)=f^{*}(x)
$$

for every $x \in G$ and every continuous function $f: M \rightarrow \mathbb{R}$.

Proof. See pp. 73 - 74 in [4].

The Birkhoff ergodic theorem has many applications in different areas of mathematics. One of them is the quantitative version of Poincaré's recurrence theorem. More precisely, we have that

Proposition 2.3.3. Let $(X, \mathcal{B}, \mu, T)$ be an ergodic system and let $E$ be a measurable subset of $X$ with positive measure $\mu(E)>0$. Then, for almost every $x \in X$, we have that

$$
\lim _{n \rightarrow \infty} \frac{\operatorname{Card}\left\{0 \leq k \leq n-1: T^{k} x \in E\right\}}{n}=\mu(E) .
$$

In other words, if $n$ is large enough then

$$
\operatorname{Card}\left\{0 \leq k \leq n-1: T^{k} x \in E\right\} \simeq n \cdot \mu(E)
$$

Proof. Let $E$ be a measurable subset of $X$. It is enough to apply the Birkhoff's ergodic theorem (Theorem 2.3.1) to the integrable function $f=\mathbb{1}_{E}$. Indeed, note that

$$
\operatorname{Card}\left\{0 \leq k \leq n-1: T^{k} x \in E\right\}=\sum_{k=0}^{n-1} \mathbb{1}_{E}\left(T^{k} x\right) .
$$

Thus, by Theorem 2.3.1, we have that

$$
\lim _{n \rightarrow \infty} \frac{1}{n} \sum_{k=0}^{n-1} \mathbb{1}_{E}\left(T^{k} x\right)=\int_{X} \mathbb{1}_{E} d \mu=\mu(E)
$$

for $\mu$-a.e. $x \in X$.

Thus, for almost every $x$ in $E$ the limit of the frequencies of visits exists and is equal to $\mu(E)$ :

$$
\lim _{n \rightarrow \infty} \frac{\operatorname{Card}\left\{0 \leq k \leq n-1: T^{k} x \in E\right\}}{n}=\mu(E) .
$$

Before we formulate Kingman's theorem, we introduce some terminology. 
Definition 2.3.1. We say that a sequence of functions $\varphi_{n}: X \rightarrow \mathbb{R}$ is subadditive with respect to the transformation $T: X \rightarrow X$ if for all (or a.e.) $x \in X$,

$$
\varphi_{m+n}(x) \leq \varphi_{m}(x)+\varphi_{n}\left(T^{m} x\right)
$$

for all $n, m \in \mathbb{N}$.

Example 2.3.1. Let $A: X \rightarrow \mathrm{SL}_{d}(\mathbb{R})$ be a continuous function and let $T: X \rightarrow X$ be a transformation. Define

$$
A^{(n)}(x):=A\left(T^{n-1} x\right) \cdots A(T x) A(x) .
$$

Then, the sequence $\varphi_{n}(x)=\log \left\|A^{(n)}(x)\right\|$ is subadditive. Indeed, note that

$$
\begin{aligned}
A^{(m+n)}(x) & =A\left(T^{n-1}\left(T^{m} x\right)\right) \cdots A\left(T^{m} x\right) A\left(T^{m-1} x\right) \cdots A(T x) A(x) \\
& =A^{(n)}\left(T^{m} x\right) \cdot A^{(m)}(x) .
\end{aligned}
$$

Hence,

$$
\begin{aligned}
\varphi_{m+n}(x) & =\log \left\|A^{(n)}\left(T^{m} x\right) \cdot A^{(m)}(x)\right\| \\
& \leq \log \left(\left\|A^{(n)}\left(T^{m} x\right)\right\| \cdot\left\|A^{(m)}(x)\right\|\right) \\
& \leq \log \left\|A^{(n)}\left(T^{m} x\right)\right\|+\log \left\|A^{(m)}(x)\right\|=\varphi_{m}(x)+\varphi_{n}\left(T^{m} x\right)
\end{aligned}
$$

for all $m, n \in \mathbb{N}$ and $x \in X$.

Theorem 2.3.4 (Kingman for measure preserving transformations). Let $(X, \mu, T)$ be a measurable preserving dynamical system. where $\mu$ is a probability measure, and let $\left\{\varphi_{n}\right\}$ be a subadditive sequence of measurable functions on $L^{1}(\mu)$.

Then, the sequence $\left(\frac{\varphi_{n}}{n}\right)$ converges to a T-invariant measurable function $\varphi: X \rightarrow[-\infty,+\infty)$ for $\mu$-almost every $x \in X$. This function $\varphi$ is such that $\varphi(x)=\inf _{n} \frac{1}{n} \varphi_{n}^{*}(x)$, where $\varphi_{n}^{*}=\int_{X} \varphi_{n} d \mu$.

Moreover, $\varphi^{+} \in L^{1}(\mu)$ and

$$
\int \varphi d \mu=\lim _{n \rightarrow \infty} \frac{1}{n} \int \varphi_{n} d \mu=\inf _{n} \frac{1}{n} \int \varphi_{n} d \mu .
$$

The proof of Katznelson and Weiss presented in [10] follows the same approach they used in the proof of Birkhoff's theorem.

As a consequence of theorem 2.3.4, there is a version of Kingman's Theorem for ergodic systems. More precisely, 
Corollary 2.3.2 (Kingman's for ergodic transformations). Let $(X, \mu, T)$ be an ergodic system and let $\left\{\varphi_{n}\right\}_{n \geq 1}$ be a sequence of subadditive measurable functions. Then, the sequence $\left(\frac{\varphi_{n}}{n}\right)$ converges to a constant $L(\varphi)$ for $\mu$-almost every $x \in X$. Furthermore, we have

$$
L(\varphi)=\lim _{n \rightarrow \infty} \frac{1}{n} \int \varphi_{n} d \mu=\inf _{n} \frac{1}{n} \int \varphi_{n} d \mu
$$

Definition 2.3.2. The constant $L(\varphi)$ on the Theorem 2.3.2 is called the (maximal) Lyapunov exponent of the subadditive process $\left\{\varphi_{n}\right\}_{n \geq 1}$.

\section{4}

\section{Uniquely Ergodic Systems}

Definition 2.4.1. We say that a measure preserving dynamical system $(X, \mu, T)$ is uniquely ergodic if $T: X \rightarrow X$ is a homeomorphism and $\mu$ is the unique $T$-invariant probability measure on $X$.

Observation 1. If $(X, \mu, T)$ is a uniquely ergodic system, then necessarily $\mu$ is ergodic. Indeed, suppose that there exists an invariant subset $A$ in $M$ with $0<\mu(A)<1$. Thus,

$$
\mu_{A}(E):=\frac{\mu(E \cap A)}{\mu(A)}
$$

is a different $T$-invariant probability measure on $X$, contradicting the hypothesis that $\mu$ is the unique $T$-invariant measure on $X$.

The following proposition gives several equivalent ways of defining unique ergodicity.

Proposition 2.4.1. Let $f: X \rightarrow X$ be a continuous map. The following conditions are equivalent:

(a) f admits a unique invariant probability measure.

(b) $f$ admits a unique ergodic probability measure.

(c) for every continuous function $\phi: X \rightarrow \mathbb{R}$, the sequence of averages along a trajectory $\frac{1}{n} \sum_{k=0}^{n-1}\left(\phi \circ f^{k}\right)(x)$ converges to a constant for almost every $x \in X$.

(d) for every continuous function $\phi: X \rightarrow \mathbb{R}$, the sequence of averages along a trajectory $\frac{1}{n} \sum_{k=0}^{n-1} \phi \circ f^{k}$ converges uniformly to a constant.

Proof. See p. 160 in [4]. 
Observe that the item (d) in Proposition 2.4.1 is a version of Birkhoff's Theorem for uniquely ergodic system. More precisely,

Theorem 2.4.2 (Birkhoff's ergodic theorem for uniquely ergodic systems). Let $(X, \mathcal{B}, \mu, T)$ be a uniquely ergodic system and let $f: X \rightarrow \mathbb{R}$ be a continuous function. Then, the convergence of the Birkhoff averages

$$
\lim \frac{1}{n} \sum_{j=0}^{n-1} f\left(T^{j} x\right)=\int_{X} f d \mu
$$

is uniform on $X$.

Observation 2. Let $\left(X, \mathcal{C}, \nu_{1}\right)$ and $\left(X, \mathcal{C}, \nu_{2}\right)$ be two probability spaces and $\sigma$ the Bernoulli shift defined in the Example 2.1.4. Thus, we may define two probability measures $\mu_{1}=\nu_{1}^{\mathbb{N}}$ and $\mu_{2}=\nu_{2}^{\mathbb{N}}$ which are $\sigma$-invariant.

Then, the Bernoulli shift is not a uniquely ergodic system.

Let us prove that other examples described earlier are uniquely ergodic.

Proposition 2.4.3. Fix $d \geq 1$ and let $\theta=\left(\theta_{1}, \ldots, \theta_{d}\right) \in \mathbb{R}^{d}$ be a rationally independent vector. Then the translation map $T_{\theta}: \mathbb{T}^{d} \rightarrow \mathbb{T}^{d}$ is uniquely ergodic.

Proof. By the Proposition 2.4.1, we just need to show that for every continuous function $\varphi: \mathbb{T}^{d} \rightarrow \mathbb{R}$, there exist $c_{\varphi} \in \mathbb{R}$ such that the sequence

$$
\varphi_{n}=\frac{1}{n} \sum_{j=0}^{n-1} \varphi \circ T_{\theta}^{j}
$$

converges to $c_{\varphi}$ for every point $x \in \mathbb{T}^{d}$.

Take $c_{\varphi}=\int \varphi d \mu$. By Birkhoff's Theorem, the $\operatorname{limit} \lim _{n \rightarrow \infty} \varphi_{n}=c_{\varphi}$ exists for almost every $x \in X$. In particular, $\lim _{n \rightarrow \infty} \varphi_{n}(x)=c_{\varphi}$ for a dense set of values $x \in \mathbb{T}^{d}$.

Let $d$ the distance on the torus $\mathbb{T}^{d}=\mathbb{R}^{d} / \mathbb{Z}^{d}$, that is, the distance between any two points on $\mathbb{T}^{d}$ is the minimum of the distance between their representatives on $\mathbb{R}^{d}$. It is clear that

$$
d\left(T_{\theta}(x), T_{\theta}(y)\right)=d(x, y), \quad \forall x, y \in \mathbb{T}^{d}
$$

So,

$$
d\left(T_{\theta}^{j}(x), T_{\theta}^{j}(y)\right)=d(x, y), \quad \forall x, y \in \mathbb{T}^{d}, \forall j \geq 1 .
$$

Since $\varphi$ is a continuous function, it follows that for any $\epsilon>0$, there exists $\delta>0$ such that $d(x, y)<\delta \Rightarrow d(\varphi(x), \varphi(y))<\epsilon$. Therefore, using (2.11), if $d(x, y)<\delta$ then $\left|\varphi\left(T_{\theta}(x)\right)-\varphi\left(T_{\theta}(y)\right)\right|<\epsilon$, for every $j \geq 0$. 
It follows that for all $x, y$ such that $d(x, y)<\delta$, we have that

$$
\begin{aligned}
\left|\varphi_{n}(x)-\varphi_{n}(y)\right| & =\left|\frac{1}{n} \sum_{j=0}^{n-1}\left[\varphi \circ T_{\theta}^{j}(x)-\varphi \circ T_{\theta}^{j}(y)\right]\right| \\
& \leq \frac{1}{n} \sum_{j=0}^{n-1}\left|\varphi \circ T_{\theta}^{j}(x)-\varphi \circ T_{\theta}^{j}(y)\right| \\
& <\frac{1}{n} \sum_{j=0}^{n-1} \epsilon=\epsilon
\end{aligned}
$$

for all $n \in \mathbb{N}$. Hence, the sequence $\left(\varphi_{n}\right)_{n \in \mathbb{N}}$ is equicontinuous.

Now, suppose that there exists $\bar{x} \in \mathbb{T}^{d}$ such that $\left(\varphi_{n}(\bar{x})\right)$ does not converges to $c_{\varphi}$. Then, there exist $c \neq c_{\varphi}$ and a subsequence $\left(n_{k}\right)$ such that $\lim _{k \rightarrow \infty} \varphi_{n_{k}}(\bar{x})=c$. We can suppose that the sequence $\left(\varphi_{n_{k}}\right)_{k}$ is uniformly convergent. Let $\psi$ such that $\lim _{k \rightarrow \infty} \varphi_{n_{k}}=\psi$. Then, $\psi$ is a continuous function such that $\psi(x)=c_{\varphi}$ for a dense subset of $\mathbb{T}^{d}$ and $\varphi(\bar{x})=c \neq c_{\varphi}$. Hence, we get a contradiction.

Lemma 2.4.4. Let $\pi: \mathbb{T}^{2} \rightarrow \mathbb{T}$ be the projection map $\pi\left(\theta_{1}, \theta_{2}\right)=\theta_{1}$. If $\mu$ is an invariant probability measure for $f$ then the projection measure $\pi_{*} \mu=\mu \circ \pi^{-1}$ coincides with the Lebesgue measure $m$ on $\mathbb{T}$.

Proof. Let $E$ be any measurable subset on $\mathbb{T}$,

$$
\left(\pi_{*} \mu\right)\left(f_{0}^{-1}(E)\right)=\mu\left(\pi^{-1} f_{0}^{-1}(E)\right)
$$

Since $\pi \circ f=f_{0} \circ \pi$ and $\mu$ is a $f$-invariant measure, we have that

$$
\mu\left(\pi^{-1} f_{0}^{-1}(E)\right)=\mu\left(f^{-1} \pi^{-1}(E)\right)=\mu\left(\pi^{-1}(E)\right)=\left(\pi_{*} \mu\right)(E) .
$$

Hence, $\left(\pi_{*} \mu\right)\left(f_{0}^{-1}(E)\right)=\left(\pi_{*} \mu\right)(E)$ for every measurable subset $E$, that is, $\pi_{*} \mu$ is an $f_{0}$-invariant probability measure. Since $f_{0}$ is uniquely ergodic, it follows that $\pi_{*} \mu$ coincides with the Lebesgue measure $m$ on $\mathbb{T}$.

Proposition 2.4.5. If $\mu$ and $\nu$ are probability measures on a metric space $M$ such that

$$
\int \varphi d \mu=\int \varphi d \nu
$$

for every bounded Lipschitz function $\varphi: M \rightarrow \mathbb{R}$, then $\mu=\nu$.

Proof. See pp.449 - 450 in [4]. 
Proposition 2.4.6. Let $f$ be the skew-translation defined in the Example 2.1.3. Then, the Lebesgue measure on $\mathbb{T}^{2}$ is uniquely ergodic for $f$.

Proof. We can rewrite $\mathbb{T}^{2}=\mathbb{T}^{1} \times \mathbb{T}^{1}$ and

$$
f: \mathbb{T}^{1} \times \mathbb{T}^{1} \rightarrow \mathbb{T}^{1} \times \mathbb{T}^{1}, \quad f\left(\theta_{1}, \theta_{2}\right)=\left(f_{0}\left(\theta_{1}\right), \theta_{1}+\theta_{2}\right)
$$

where $f_{0}\left(\theta_{1}\right)=\theta_{1}+\alpha$. By Proposition 2.4.3, the transformation

$$
f_{0}: \mathbb{T}^{1} \rightarrow \mathbb{T}^{1}
$$

is uniquely ergodic.

Now, suppose that there is an $f$-invariant probability measure $\mu$ which is also an ergodic measure for $f$. Let $G(\mu)$ be a subset of $\mathbb{T}^{2}$ where $G(\mu)$ is a set of values $\theta \in \mathbb{T}^{2}$ such that

$$
\lim \frac{1}{n} \sum_{j=0}^{n-1} \varphi\left(f^{j} x\right)=\int_{X} \varphi d \mu \text { for every continuous function } \varphi: \mathbb{T}^{2} \rightarrow \mathbb{R}
$$

By Theorem 2.3.2 and the ergodicity of skew-translation, $G(\mu)$ has total measure. Define

$$
G_{0}(\mu)=\left\{\theta \in \mathbb{T}: G(\mu) \cap\left(\{\theta\} \times S^{1}\right) \neq \emptyset\right\}
$$

In other words, $G_{0}(\mu)=\pi(G(\mu))$. It is clear that $\pi^{-1}\left(G_{0}(\mu)\right)$ contains $G(\mu)$ and, then, $\pi^{-1}\left(G_{0}(\mu)\right)$ has total measure.

By Lemma 2.4.4,

$$
m\left(G_{0}(\mu)\right)=\left(\pi_{*} \mu\right)\left(G_{0}(\mu)\right)=\mu\left(\pi^{-1}\left(G_{0}(\mu)\right)\right)=1 .
$$

Similarly,

$$
m\left(G_{0}(m)\right)=\left(\pi_{*} m\right)\left(G_{0}(m)\right)=m\left(\pi^{-1}\left(G_{0}(m)\right)\right)=1 .
$$

A consequence of the above equalities is that the intersection of $G_{0}(\mu)$ and $G_{0}(m)$ has total measure. In particular, theses subsets can not be disjoint. Let $\theta_{0} \in G_{0}(\mu) \cap G_{0}(m)$. By definition, $G(\mu) \cap\left(\left\{\theta_{0}\right\} \times S^{1}\right) \neq \emptyset$. On the other hand, we can prove that $G(\mu)$ contains $\left\{\theta_{0}\right\} \times S^{1}$.

Hence, $G(\mu)$ and $G(m)$ intersect at some point of $\left\{\theta_{0}\right\} \times S_{1}$. By the definition of $G(\mu)$, this implies that the two measures $\mu$ and $m$ have the same 
integral for every continuous function. By Proposition 2.4.5, $\mu=m$, which is the conclusion we were looking for. 


\section{3}

\section{Furman's Theorem}

In section 2.3 of chapter 2, we described a version of Birkhoff's Theorem for uniquely ergodic systems. In this chapter, we answer the following question: is there an analogous result for Kingman's theorem? This question was posed by Furstenberg and answered by Furman.

Hence, the goal of this chapter is to formulate and to prove the results obtained by Furman in [6]. We begin with some preliminaries (see section 3.1) then present Furman's Theorem in section 3.2.

At the end of this chapter (see section 3.3), we also present some extensions and applications of this result to linear cocycles. They were obtained by S. Jitomirskaya and R. Mavi in [7].

\section{1}

\section{Preliminaries}

Let $(X, \mathcal{B}, \mu, T)$ be an ergodic system and let $\mathrm{SL}_{d}(\mathbb{R})$ be the linear group of invertible $d \times d$ matrices with real coefficients and determinant 1 .

Definition 3.1.1. A linear cocycle of $(X, \mathcal{B}, \mu, T)$ is a skew-product map

$$
\begin{aligned}
F_{A}: X \times \mathbb{R}^{d} & \rightarrow X \times \mathbb{R}^{d} \\
(x, v) & \mapsto(T x, A(x) v)
\end{aligned}
$$

where $A: X \rightarrow \mathrm{SL}_{d}(\mathbb{R})$ is a measurable map.

Define

$$
A^{(n)}(x)=A\left(T^{n-1} x\right) \cdots A(T x) A(x) .
$$

It is easy to verify that the iterates of $F_{A}$ are given by:

$$
F_{A}^{n}(x, v)=\left(T^{n} x, A^{(n)}(x) v\right)
$$

Definition 3.1.2. Let $M$ be a metric space. We say that a subset $E$ on $M$ is an $F_{\sigma}$ set if $E=\bigcup_{k \in \mathbb{N}} F_{k}$, where $F_{k}$ is a closed set in $M$ for all $k \in \mathbb{N}$. 
Definition 3.1.3. Let $A$ and $B$ be disjoint closed subsets of a topological space $X$.

a) We say that $A$ and $B$ are separated by neighbourhoods if there are neighbourhoods $U$ of $A$ and $V$ of B such that $U \cap V=\emptyset$.

b) We say that $A$ and $B$ are separated by a function if there exists a continuous function $f: X \rightarrow[0,1]$ such that $f(a)=0$ for every $a \in A$ and $f(b)=1$ for every $b \in B$.

Definition 3.1.4. We say that a topological space $X$ is a normal space if any two disjoint closed subsets of $X$ can be separeted by neighbourhoods.

Example 3.1.1. A metric space is a normal space.

Lemma 3.1.1 (Urysohn). A topological space $X$ is a normal space if, and only if, any two disjoint closed subsets of $X$ can be separated by a continuous function.

Proof. See p. 115 in [11].

Definition 3.1.5. Let $\left(X, d_{1}\right)$ and let $\left(X, d_{2}\right)$ be metric spaces. We say that $f: X \rightarrow Y$ is a uniformly continuous function if

$$
\forall \epsilon>0, \exists \delta>0 ; \forall x, y \in X, d_{1}(x, y)<\delta \Rightarrow d_{2}(f(x), f(y))<\epsilon
$$

Definition 3.1.6. Let $M$ be a metric space. We say that $f: M \rightarrow \mathbb{R}$ is an upper semicontinuous function at a point $x \in M$ if $\limsup _{y \rightarrow x} f(y) \leq f(x)$, that is,

$$
\forall \epsilon>0, \exists \delta>0 ;|y-x|<\delta \Rightarrow f(y) \leq f(x)+\epsilon \text {. }
$$

Observation 3. A function $f: M \rightarrow \mathbb{R}$ is upper semicontinuous if and only if $f^{-1}(-\infty, c)$ is an open subset of $M$, for every $c \in \mathbb{R}$.

Proposition 3.1.2. Let $(M, d)$ be a metric space and let $\left\{f_{n}\right\}$ be a sequence of upper semicontinuous functions on $M$. Define the function $f: M \rightarrow \mathbb{R}$ such that $f(a):=\inf _{n \geq 1} f_{n}(a)$ is the pointwise infimum of these functions. Then, $f$ is also an upper semicontinuous function.

Proof. We need to show that the set $f^{-1}(-\infty, c)$ is a open subset of $M$, for every $c \in \mathbb{R}$. Note that

$$
f(x)<c \Leftrightarrow \inf _{n \geq 1} f_{n}(x)<c \Leftrightarrow \exists n \in \mathbb{N} ; f_{n}(x)<c \Leftrightarrow \exists n \in \mathbb{N} ; x \in f_{n}^{-1}(-\infty, c) .
$$


Hence, $f^{-1}(-\infty, c)=\bigcup_{n \in \mathbb{N}} f_{n}^{-1}(-\infty, c)$.

By hypothesis, $f_{n}$ is an upper semicontinuous function for every $n \in \mathbb{N}$, so $f_{n}^{-1}(-\infty, c)$ is an open subset of $M$.

Hence, $f_{n}^{-1}(-\infty, c)$ is also an open subset of $M$.

\section{2}

\section{On the subadditive ergodic theorem for uniquely ergodic systems}

In this section, we formulate and prove Furman's theorem regarding the convergence in Kingman's subadditive ergodic theorem for a continuous subadditive process on a compact, uniquely ergodic system.

Let us recall that a subadditive process on $(X, \mu, T)$ is a sequence $\left\{f_{n}\right\}$ of measurable functions such that

$$
f_{n+m} \leq f_{n}+f_{m} \circ T^{n}, \quad \forall n, m \in \mathbb{N} .
$$

Throughout this section, unless otherwise noted, we will assume that $X$ is a compact metric space.

Theorem 3.2.1 (Furman). Let $(X, \mu, T)$ be an uniquely ergodic system, where $X$ is a compact metric space. Let $\left\{f_{n}\right\}$ be a subadditive process on $X$, where each function $f_{n}$ is continuous. Then, for every $x \in X$,

$$
\limsup _{n \rightarrow \infty} \frac{1}{n} f_{n}(x) \leq L(f)
$$

uniformly on $X$. That is, for every $\epsilon>0$, there exists $N \in \mathbb{N}$ such that

$$
\frac{1}{n} f_{n} \leq L(f)+\epsilon, \forall n \geq N, \forall x \in X
$$

However, for any $F_{\sigma}$ set $E$ with $\mu(E)=0$, there exists a continuous subadditive sequence $\left\{f_{n}\right\}$ on $X$, such that

$$
\lim \sup \frac{1}{n} f_{n}(x)<L(f), \quad \forall x \in E
$$

Proof. Fix $\epsilon>0$. By Theorem 2.3.2, for $\mu$-a.e. $x \in X$, there exists $n \in \mathbb{N}$ such that

$$
\frac{1}{n} f_{n}(x)-\epsilon<L(f)
$$

Let $n(x)$ be the first $n$ such that the inequality (3.1) is satisfied. That is, 
for $\mu$-a.e. $x \in X$, define

$$
n(x)=\inf \left\{n \in \mathbb{N} ; f_{n}(x)<n(L(f)+\epsilon)\right\} .
$$

On the remaining set of measure 0 , we may put $n(x)=1$.

For each $N \in \mathbb{N}$, define the set

$$
\mathcal{A}_{N}=\{x \in X ; n(x) \leq N\}=\bigcup_{n=1}^{N}\left\{x \in X ; f_{n}(x)<n \cdot(L(f)+\epsilon)\right\}
$$

Hence, $\mathcal{A}_{N} \nearrow X$ since for each $x \in X, n(x) \in \mathbb{N}$ and $\mu\left(\mathcal{A}_{N}\right) \rightarrow \mu(X)=1$.

Fix $N \in \mathbb{N}$ such that $\mu\left(X \backslash \mathcal{A}_{N}\right)<\epsilon$.

For every $x \in X$ we define a sequence of indices $\left\{n_{j}\right\}$ and points $\left\{x_{j}\right\}$ by the following rule:

$$
x_{1}=x, \quad \text { and } \quad n_{1}= \begin{cases}n\left(x_{1}\right), & \text { if } x_{1} \in \mathcal{A}_{N} \\ 1, & \text { if } x_{1} \notin \mathcal{A}_{N}\end{cases}
$$

For $j \geq 1$, define

$$
x_{j+1}=T^{n_{j}} x_{j}=T^{n_{1}+\cdots+n_{j}} x, \quad \text { and } \quad n_{j+1}= \begin{cases}n\left(x_{j+1}\right), & \text { if } x_{j+1} \in \mathcal{A}_{N} \\ 1, & \text { if } x_{j+1} \notin \mathcal{A}_{N}\end{cases}
$$

Let $M>N \cdot\left\|f_{1}\right\|_{\infty} / \epsilon$. Since $1 \leq n_{j} \leq N$, it follows that the sequence $a_{n}=\sum_{j=1}^{n} n_{j}$ is such that $a_{n} \nearrow \infty$. Hence, there exists $p \in \mathbb{N}$ such that

$$
n_{1}+\cdots+n_{p-1} \leq M \leq n_{1}+\cdots+n_{p}
$$

that is, there exists $K \in\left\{0, \ldots, n_{p}\right\}$ such that $M=n_{1}+\cdots+n_{p-1}+K$.

Observe that

$$
\begin{aligned}
f_{K}\left(x_{p}\right) & =\underbrace{f_{1+\cdots+1}^{1+\cdots}}_{K \text { times }}\left(x_{p}\right) \leq f_{K-1}\left(x_{p}\right)+f_{1}\left(T^{K-1} x_{p}\right) \\
& \leq f_{1}\left(x_{p}\right)+f_{1} \circ T\left(x_{p}\right)+\cdots+f_{1} \circ T^{K-1}\left(x_{p}\right) \\
& \leq \underbrace{\left\|f_{1}\right\|_{\infty}+\cdots+\left\|f_{1}\right\|_{\infty}}_{K \text { times }} \leq N\left\|f_{1}\right\|_{\infty}
\end{aligned}
$$

since $K \leq n_{p} \leq N$. By subadditivity,

$$
\begin{aligned}
f_{M}(x) & =f_{\left[\left(n_{1}+\cdots+n_{p-1}\right)+K\right]}(x) \\
& \leq f_{n_{1}+\cdots+n_{p-1}}(x)+f_{K}\left(T^{n_{1}+\cdots+n_{p-1}} x\right)=f_{n_{1}+\cdots+n_{p-1}}(x)+f_{K}\left(x_{p}\right) .
\end{aligned}
$$


On the other hand,

$$
\begin{aligned}
f_{\left(n_{1}+\cdots+n_{p-1}\right)}(x) & \leq f_{n_{1}+\cdots+n_{p-2}}(x)+f_{n_{p-1}}\left(T^{n_{1}+\cdots+n_{p-2}} x\right) \\
& \leq f_{\left(n_{1}+\cdots+n_{p-2}\right)}(x)+f_{n_{p-1}}\left(x_{p-1}\right) .
\end{aligned}
$$

Inductively, we have

$$
f_{M}(x) \leq \sum_{j=1}^{p-1} f_{n_{j}}\left(x_{j}\right)+f_{K}\left(x_{p}\right) \leq \sum_{j=1}^{p-1} f_{n_{j}}\left(x_{j}\right)+N \cdot\left\|f_{1}\right\|_{\infty} .
$$

Observe that

$$
f_{n_{j}}\left(x_{j}\right) \leq n_{j}(L(f)+\epsilon), \quad \text { if } x_{j} \in \mathcal{A}_{N}
$$

but, if $x_{j} \notin \mathcal{A}_{N}$, then $n_{j}=1$ and $f_{n_{j}}\left(x_{j}\right)=f_{1}\left(x_{j}\right) \leq\left\|f_{1}\right\|_{\infty}$. Hence,

$$
f_{n_{j}}\left(x_{j}\right) \leq n_{j}(L(f)+\epsilon) \cdot \mathbb{1}_{\mathcal{A}_{N}}\left(x_{j}\right)+\left\|f_{1}\right\|_{\infty} \cdot \mathbb{1}_{X \backslash \mathcal{A}_{N}}\left(x_{j}\right) .
$$

Then, we obtain

$$
\begin{aligned}
\frac{1}{M} f_{M}(x) & \leq \frac{1}{M} \sum_{j=1}^{p-1} f_{n_{j}(x)}\left(x_{j}\right)+\frac{N}{M} \cdot\left\|f_{1}\right\|_{\infty} \\
& \left.\leq \frac{1}{M} \sum_{j=1}^{p-1}\left[n_{j}(L(f))+\epsilon\right) \cdot \mathbb{1}_{\mathcal{A}_{N}}\left(x_{j}\right)+\left\|f_{1}\right\|_{\infty} \cdot \mathbb{1}_{X \backslash \mathcal{A}_{N}}\left(x_{j}\right)\right]+\frac{N}{M}\left\|f_{1}\right\|_{\infty} \\
& \leq \frac{1}{M} \sum_{j=1}^{p-1}\left(n_{j}(L(f))+\epsilon\right)+\left\|f_{1}\right\|_{\infty} \cdot \frac{1}{M} \sum_{j=1}^{p-1} \mathbb{1}_{X \backslash \mathcal{A}_{N}}\left(x_{j}\right)+\frac{N}{M}\left\|f_{1}\right\|_{\infty} \\
& \leq(L(f)+\epsilon)+\left\|f_{1}\right\|_{\infty} \cdot \frac{1}{M} \sum_{j=1}^{M} \mathbb{1}_{X \backslash \mathcal{A}_{N}}\left(T^{j} x\right)+\frac{N}{M}\left\|f_{1}\right\|_{\infty} \\
& \leq(L(f)+\epsilon)+\left\|f_{1}\right\|_{\infty} \cdot \frac{1}{M} \sum_{j=1}^{M} \mathbb{1}_{X \backslash \mathcal{A}_{N}}\left(T^{j} x\right)+\epsilon
\end{aligned}
$$

It remains to estimate

$$
\frac{1}{M} \sum_{j=1}^{M} \mathbb{1}_{X \backslash \mathcal{A}_{N}}\left(T^{j} x\right)
$$

We claim that for $M$ sufficiently large, the summand $\frac{1}{M} \sum_{j=1}^{M} \mathbb{1}_{X \backslash \mathcal{A}_{N}}\left(T^{j} x\right)$ 
is uniformly bounded by $O(\epsilon)$. Indeed, note that

$$
\begin{aligned}
X \backslash \mathcal{A}_{N} & =\{x \in X ; n(x)>N\} \\
& =\bigcap_{n=1}^{N}\left\{x \in X ; \frac{1}{n} f_{n}(x) \geq L(f)+\epsilon\right\} .
\end{aligned}
$$

Since $f_{n}$ is a continuous function for every $n \in \mathbb{N}$ and since $X$ is a compact metric space, $\left\{x \in X ; \frac{1}{n} f_{n}(x) \geq L(f)+\epsilon\right\}$ is a closed subset of $X$ and, consequently, $X \backslash \mathcal{A}_{N}$ is also a closed subset of $X$. Hence, by the regularity of the Borel measure, there exists an open subset $U$ of $X$ such that $X \backslash A_{N} \subset U$ and $\mu(U)<\mu\left(X \backslash A_{N}\right)+\epsilon$.

Define $L=X \backslash U$. Then, by Lemma 3.1.1, there exists a continuous function $g: X \rightarrow[0,1]$ such that $\left.g\right|_{L}=0$ and $\left.g\right|_{X \backslash \mathcal{A}_{N}}=1$.

Since $\mu\left(X \backslash A_{N}\right)<\epsilon$, we have that

$$
\int_{X} g d \mu=\int_{L} g d \mu+\int_{U} g d \mu \leq \mu(U) \leq \mu\left(X \backslash \mathcal{A}_{N}\right)+\epsilon \leq 2 \epsilon .
$$

Then, for $M$ sufficiently large, and by Theorem 2.4.2,

$$
\frac{1}{M} \sum_{j=1}^{M} \mathbb{1}_{X \backslash \mathcal{A}_{N}}\left(T^{j} x\right) \leq \frac{1}{M} \sum_{j=1}^{M} g\left(T^{j} x\right) \leq \int g d \mu+\epsilon \leq 3 \epsilon .
$$

Combining all of the above, we conclude that for $M$ sufficiently large and for each $x \in X$, we have that

$$
\frac{1}{n} f_{n}(x) \leq L(f)+O(\epsilon)
$$

for all $n>M$. This proves the first statement of the theorem

For the second statement, let $E=\bigcup_{k \geq 1} E_{k} \subset X$, where $E_{k}$ is a closed subset of $X$ and $\mu\left(E_{k}\right)=0$, for every $k \in \mathbb{N}$. By Lemma 3.1.1, there exists a continuous function $g_{k}: X \rightarrow[0,1]$ such that $g_{k} \uparrow_{E_{k}}=1$ and

$$
\mu\left(g_{k}\right)=\int_{X} g_{k} d \mu<2^{-k-2} .
$$

For each $n \in \mathbb{N}$, define

$$
f_{n}(x)=-\sum_{j=0}^{n-1} \sum_{k=0}^{n-1} g_{k}\left(T^{j} x\right)
$$

We note that $f_{n}$ is a continuous function and $\left\{f_{n}\right\}$ is a subadditive 
sequence. Indeed,

i) $f_{m+n}(x)=-\sum_{j=0}^{m+n-1} \sum_{k=0}^{m+n-1} g_{k}\left(T^{j} x\right)$

ii) $f_{m}(x)=-\sum_{j=0}^{m-1} \sum_{k=0}^{m-1} g_{k}\left(T^{j} x\right)$

iii) $f_{n}\left(T^{m} x\right)=-\sum_{j=0}^{n-1} \sum_{k=0}^{n-1} g_{k}\left(T^{m+j} x\right)=-\sum_{l=m}^{m+n-1} \sum_{k=0}^{n-1} g_{k}\left(T^{l} x\right)$

But,

$$
\begin{aligned}
\sum_{j=0}^{m-1} \sum_{k=0}^{m-1} g_{k}\left(T^{j} x\right)+\sum_{j=m}^{m+n-1} \sum_{k=0}^{n-1} g_{k}\left(T^{j} x\right) & \leq \sum_{j=0}^{m-1} \sum_{k=0}^{m+n-1} g_{k}\left(T^{j} x\right)+\sum_{j=m}^{m+n-1} \sum_{k=0}^{m+n-1} g_{k}\left(T^{j} x\right) \\
& =\sum_{j=0}^{m+n-1} \sum_{k=0}^{m+n-1} g_{k}\left(T^{j} x\right) .
\end{aligned}
$$

Hence,

$$
f_{m+n}(x) \leq f_{m}(x)+f_{n}\left(T^{m} x\right)
$$

On the other hand,

$$
\begin{aligned}
\lim _{n \rightarrow \infty} \frac{1}{n} \int f_{n} d \mu & =\lim _{n \rightarrow \infty} \frac{1}{n} \int-\sum_{j=0}^{n-1} \sum_{k=0}^{n-1} g_{k}\left(T^{j} x\right) \\
& =-\lim _{n \rightarrow \infty} \frac{1}{n} \sum_{j=0}^{n-1} \sum_{k=0}^{n-1} \int g_{k}\left(T^{j} x\right) \\
& \geq-\lim _{n \rightarrow \infty} \frac{1}{n} \sum_{j=0}^{n-1} \sum_{k=0}^{n-1} \frac{1}{2^{k+2}} \\
& =-\lim _{n \rightarrow \infty} \frac{1}{n} \cdot n \sum_{k=0}^{n-1} \frac{1}{2^{k+2}} \\
& =-\sum_{n=0}^{\infty} \frac{1}{2^{n+2}} .
\end{aligned}
$$

Then, by Theorem 2.3.2,

$$
L(f)=\lim _{n \rightarrow \infty} \frac{1}{n} \int f_{n} d \mu \geq-\sum_{n=0}^{\infty} \frac{1}{2^{n+2}}=-\frac{1}{2} .
$$

But, for any $x \in E$, we have

$$
\limsup _{n \rightarrow \infty} \frac{1}{n} f_{n}(x)<-1<-\frac{1}{2} \leq L(f) .
$$

Hence, this proves the second statement of the theorem. 
Corollary 3.2.1. Let $(X, \mu, T)$ be a compact, uniquely ergodic system and let $A: X \rightarrow \mathrm{SL}_{d}(\mathbb{R})$ be a continuous function. Then, for every $x \in X$

$$
\limsup _{n \rightarrow \infty} \frac{1}{n} \log \left\|A^{(n)}(x)\right\| \leq L(f)
$$

uniformly on $X$.

Proof. Take $f_{n}(x)=\log \left\|A^{(n)}(x)\right\|$. By Example 2.3.1, $\left\{f_{n}\right\}$ is a subadditive sequence. Then, applying Theorem 3.2.1, we have the inequality desired.

Observation 4. By Theorem 3.2.1, we conclude that there exists a continuous, subadditive sequence $\left\{f_{n}\right\}$ such that $\left(\frac{f_{n}}{n}\right)$ does not converge to $L(f)$ uniformly on $X$.

\section{3}

\section{Uniform upper semicontinuity of the Lyapunov exponent}

Let $(X, \mu, T)$ be an ergodic Borel probability space and let $\Gamma(X)$ be the space of all $T$-subadditive sequences $f=\left\{f_{n}\right\}$, where $f_{n}$ is a continuous function for all $n \in \mathbb{N}$.

By Theorem 2.3.2 we have that for almost every $x \in X$,

$$
L(f)=\lim _{n \rightarrow \infty} \frac{1}{n} \int f_{n} d \mu=\inf _{n} \frac{1}{n} \int f_{n} d \mu .
$$

$L(f)$ is called the Lyapunov exponent of the process $f=\left\{f_{n}\right\}$.

Define $d: \Gamma(X) \times \Gamma(X) \rightarrow \mathbb{R}$ such that

$$
d(g, f)=\sum_{n \geq 1} \frac{1}{2^{n}} \frac{\left\|g_{n}-f_{n}\right\|_{\infty}}{1+\left\|g_{n}-f_{n}\right\|_{\infty}}
$$

where

$$
\|f\|_{\infty}=\max _{\theta \in X}|f(\theta)| .
$$

It is easy to verify that $(\Gamma(X), d)$ is a metric space.

Proposition 3.3.1. Let $(X, \mu, T)$ be an ergodic system, where $X$ is a compact metric space, and let $f=\left\{f_{n}\right\} \in \Gamma(X)$. Then, the Lyapunov exponent function $L: \Gamma(X) \rightarrow \mathbb{R}$ is upper semicontinuous with respect to the metric $d$.

Proof. We claim that $\{f\} \mapsto \frac{1}{n} \int_{X} f_{n} d \mu$ is a continuous map for each $n \in \mathbb{N}$. Indeed, fix $n \in \mathbb{N}$. 
Let $\epsilon>0$. Then for $\delta=\frac{\epsilon n}{2^{n}(1+\epsilon n)}$, it is easy to see that

$$
d(g, f)<\delta \Rightarrow\left\|g_{n}-f_{n}\right\|_{\infty}<\epsilon n
$$

Hence,

$$
\left|\frac{1}{n} \int_{X} f_{n} d \mu-\frac{1}{n} \int_{X} g_{n} d \mu\right| \leq \frac{1}{n} \int_{X}\left\|f_{n}-g_{n}\right\|_{\infty} d \mu<\frac{\epsilon n}{n}=\epsilon
$$

which proves our claim.

Then, by the arbitrariness of $n,\{f\} \rightarrow \frac{1}{n} \int_{X} f_{n} d \mu$ is a continuous map on $(\Gamma(X), d)$, for every $n \in \mathbb{N}$. Consequently, by Proposition 3.1.2,

$$
\{f\} \mapsto \inf _{n} \frac{1}{n} \int_{X} f_{n} \mu(d x)=L(f)
$$

is a upper semicontinuous map in $(\Gamma(x), d)$.

Combining these properties with Theorem 3.2.1, we obtain the following result due to S. Jitomirskaya and R. Mavi (see [7]).

Theorem 3.3.2. Let $(X, \mu, T)$ be a compact, uniquely ergodic system and let $f=\left\{f_{n}\right\} \in \Gamma(X)$. Then, the Lyapunov exponent function $L: \Gamma(X) \rightarrow \mathbb{R}$ is uniformly upper semicontinuous with respect to the metric $d$. That is, given $\epsilon>0$, there exist $\delta_{\epsilon}, n_{\epsilon}$, such that for all $g=\left\{g_{n}\right\} \in \Gamma(X)$ with $d(f, g)<\delta_{\epsilon}$, for all $n>n_{\epsilon}$ and for all $x \in X$, we have

$$
\frac{1}{n} g_{n}(x) \leq L(f)+\epsilon
$$

Proof. Fix $\epsilon$. By Corollary 2.3.2, there exists $n_{0} \in \mathbb{N}$ such that

$$
\frac{1}{n_{0}} \int_{X} f_{n_{0}}(x) d \mu<L(f)+\epsilon
$$

By Theorem 2.4.2 and Theorem 3.2.1, there exists $m \in \mathbb{N}$ such that for every $x \in X$,

$$
\frac{1}{m} \sum_{k=0}^{m-1} f_{n_{0}}\left(T^{k n} x\right)<\int_{X} f_{n_{0}}(x) d \mu(d x)+\epsilon<n_{0}(L(f)+2 \epsilon) .
$$

Take $M=n_{0} m$. Since $\left\{f_{n}\right\}_{n \in \mathbb{N}}$ is a subadditive sequence,

$$
f_{M}(x)=f_{n_{0} m}(x) \leq f_{n_{0}}(x)+f_{(m-1) n_{0}}\left(T^{n_{0}} x\right) \leq f_{n_{0}}(x)+f_{n_{0}}\left(T^{n_{0}} x\right)+f_{(m-2) n_{0}}\left(T^{2 n_{0}} x\right) .
$$


Hence, by induction and by the inequality (3.2), we have that

$$
\frac{1}{M} f_{M}(x) \leq \frac{1}{M} \sum_{k=0}^{m-1} f_{n_{0}}\left(T^{k n_{0}} x\right)<L(f)+2 \epsilon
$$

for every $x \in X$.

As in the proof of Proposition 3.3.1, since $M$ is fixed, there is $\delta_{\epsilon}>0$ such that if $d(f, g)<\delta_{\epsilon}$ then $\left\|g_{M}-f_{M}\right\|_{\infty}<\epsilon$. Then in particular, for all $x \in X$,

$$
\frac{1}{M} g_{M}(x)<\frac{1}{M} f_{M}(x)+\frac{\epsilon}{M}<L(f)+2 \epsilon+\frac{\epsilon}{M}<L(f)+3 \epsilon .
$$

Put $C:=\sup _{x \in X}\left|g_{1}(x)\right|<\infty$, since $g_{1}$ is continuous and $X$ is compact.

Let $n \geq M$. Then, there are $k \in \mathbb{N}$ and $r \in\{0, \ldots, M-1\}$ such that $n=k M+r$. By subadditivity, we have

$$
\begin{aligned}
g_{k M+r}(x) & \leq g_{M}(x)+g_{(k-1) M+r}\left(T^{M} x\right) \\
& \leq g_{M}(x)+g_{M}\left(T^{M} x\right)+g_{(k-2) M+r}\left(T^{2 M} x\right) .
\end{aligned}
$$

Then, by induction and using (3.3),

$$
g_{k M+r}(x) \leq \sum_{l=1}^{k} g_{M}\left(T^{l M} x\right)+g_{r}\left(T^{k M} x\right) \leq k M(L(f)+3 \epsilon)+C r .
$$

Divide both sides by $n=k M+r$ to get:

$$
\frac{1}{n} g_{n}(x) \leq L(f)+3 \epsilon+\frac{C r}{k M+r}<L(f)+4 \epsilon
$$

since $n$ is large enough.

This completes the proof.

Proposition 3.3.3. Let

$$
\mathcal{C}(X)=\left\{A: X \rightarrow \mathrm{SL}_{d}(\mathbb{R}): A \text { continuous }\right\}
$$

endowed with any distance dist such that $\operatorname{dist}(A, B) \geq\|A-B\|$.

Given $A \in \mathcal{C}(X)$ and $n \in \mathbb{N}$, define $f_{n}^{A}=\log \left\|A^{(n)}\right\|$, and denote by $f^{A}:=\left\{f_{n}^{A}\right\}_{n \in \mathbb{N}}$ the corresponding subadditive sequence.

Then the function

$$
(\mathcal{C}(X), \text { dist }) \ni A \rightarrow f_{A} \in(\Gamma(X), d)
$$

is uniformly continuous. 
Proof. Let $\epsilon>0$. Since $\sum_{n=1}^{\infty} \frac{1}{2^{n}}<\infty$, there exists $N \in \mathbb{N}$ such that

$$
\sum_{n=N+1}^{\infty} \frac{1}{2^{n}}<\frac{\epsilon}{2}
$$

Take $\delta=\frac{\epsilon}{N}$ and take $A, B \in \mathcal{C}(X)$ such that $\operatorname{dist}(A, B)<\delta$. Then, $\|A-B\|_{\infty}<\delta$. By the mean value theorem, there exists $c \in \mathbb{R}$ such that $\min \left\{\left\|A^{(n)}\right\|,\left\|B^{(n)}\right\|\right\} \leq c \leq \max \left\{\left\|A^{(n)}\right\|,\left\|B^{(n)}\right\|\right\}$ and

$$
\begin{aligned}
\left|f_{n}^{A}-f_{n}^{B}\right| & =\left|\log \left\|A^{(n)}\right\|-\log \left\|B^{(n)}\right\|\right|=\frac{\left\|A^{(n)}\right\|-\left\|B^{(n)}\right\| \mid}{c} \\
& \leq \frac{\|\| A^{(n)}\|-\| B^{(n)} \| \mid}{\min \left\{\left\|A^{(n)}\right\|,\left\|B^{(n)}\right\|\right\}} \\
& \leq\left\|A^{(n)}-B^{(n)}\right\| .
\end{aligned}
$$

Here we used the fact that if a matrix $g$ has determinant 1, then its operator norm $\|g\| \geq 1$.

On the other hand, note that

$$
\begin{aligned}
\left\|A^{(2)}-B^{(2)}\right\| & =\|A(T x) A(x)-B(T x) B(x)\| \\
& \leq\|A(T x)\|\|A(x)-B(x)\|+\|A(T x)-B(T x)\|\|B(x)\| \\
& \leq 2 c \delta
\end{aligned}
$$

where $c=\max \{\|A\|,\|B\|\}$.

By induction, it is easy to show that for all $n \geq 1,\left\|A^{(n)}-B^{(n)}\right\| \leq n c^{n-1} \delta$.

Therefore,

$$
\left|f_{n}^{A}-f_{n}^{B}\right| \leq n c^{n-1} \delta .
$$

Observe that

$$
\frac{\left|f_{n}^{A}-f_{n}^{B}\right|}{1+\left|f_{n}^{A}-f_{n}^{B}\right|} \leq 1 \Rightarrow \sum_{n=N}^{\infty} \frac{1}{2^{n}} \frac{\left|f_{n}^{A}-f_{n}^{B}\right|}{1+\left|f_{n}^{A}-f_{n}^{B}\right|} \leq \sum_{n=N}^{\infty} \frac{1}{2^{n}}<\frac{\epsilon}{2}
$$


Hence, since $\delta \leq \epsilon / n$ and $1+\left|f_{n}^{A}-f_{n}^{B}\right| \geq 1, \forall 1 \leq n \leq N$, we have that

$$
\begin{aligned}
d\left(f^{A}, f^{B}\right) & =\sum_{n=1}^{\infty} \frac{1}{2^{n}} \frac{\left|f_{n}^{A}-f_{n}^{B}\right|}{1+\left|f_{n}^{A}-f_{n}^{B}\right|} \\
& =\sum_{n=1}^{N} \frac{1}{2^{n}} \frac{\left|f_{n}^{A}-f_{n}^{B}\right|}{1+\left|f_{n}^{A}-f_{n}^{B}\right|}+\sum_{n=N+1}^{\infty} \frac{1}{2^{n}} \frac{\left|f_{n}^{A}-f_{n}^{B}\right|}{1+\left|f_{n}^{A}-f_{n}^{B}\right|} \\
& \leq \sum_{n=1}^{N} \frac{1}{2^{n}} \frac{n c^{n-1} \delta}{1+\left|f_{n}^{A}-f_{n}^{B}\right|}+\frac{\epsilon}{2} \\
& \leq \epsilon\left(\sum_{n=1}^{N} \frac{c^{n-1}}{2^{n}}\right)+\frac{\epsilon}{2} \\
& =\epsilon\left(\frac{1-2^{N} c^{N}}{2^{N} c^{N-1}(1-2 c)}\right)+\frac{\epsilon}{2}=O(\epsilon) .
\end{aligned}
$$

Since $\epsilon$ was arbitrary, we conclude that $(\mathcal{C}(X)$, dist $) \rightarrow(\Gamma(X), d)$ is a uniformly continuous map.

Corollary 3.3.1. Let $(X, \mu, T)$ be a compact, uniquely ergodic system. Then, given $A: X \rightarrow \mathrm{SL}_{d}(\mathbb{R})$ and $\epsilon>0$, there exist $n_{\epsilon} \in \mathbb{N}$ and $\delta_{\epsilon}>0$ such that for every $B: X \rightarrow \mathrm{SL}_{d}(\mathbb{R})$ with $\operatorname{dist}(B, A)<\delta_{\epsilon}$, for all $n \geq n_{\epsilon}$ and for all $x \in X$,

$$
\frac{1}{n} \log \left\|B^{(n)}(x)\right\|<L(A)+\epsilon
$$


4

\section{A convergence rate for Birkhoff sums of Diophantine torus translations}

In this chapter we answer the second question formulated in the introduction: can we estimate the convergence rate of the Birkhoff averages for certain types of uniquely ergodic systems and observables?

We first introduce the relevant concepts, formulate the main theorem and describe some related results (Section 4.1). In Section 4.2 we review some basic facts about continued fractions, then we discuss the concept of Diophantine condition following [12] (Section 4.3). In Section 4.4 we present an intuitive idea of the proof of the rate of convergence result for Diophantine translations on the torus with Hölder observables, then we give a formal proof in Section 4.5. We end the chapter with the description of some related future problems.

Throughout this chapter, we denote by $\mathcal{T}_{n}$ the set of trigonometric polynomials of degree $\leq n$.

Moreover, if $x \in \mathbb{R}$, we use the notation $e(x)=e^{2 \pi i x} \in S^{1}$, where $S^{1}$ is the unit circle regarded as a subset of the complex plane $\mathbb{C}$.

\section{1}

\section{Introduction and statement}

Let us recall that for a uniquely ergodic system, given a continuous observable, the corresponding Birkhoff averages converge everywhere and uniformly.

A natural question is then: can we estimate the convergence rate of the Birkhoff averages for certain types of uniquely ergodic systems and observables?

We obtain a positive answer to this question in the case of a Diophantine torus translation with a Hölder continuous observable.

Let $\mathbb{T}=\mathbb{R} / \mathbb{Z}$ be the one dimensional torus endowed with the Lebesgue measure and let $T: \mathbb{T} \rightarrow \mathbb{T}, T x=x+\omega$ be the translation on $\mathbb{T}$ by an irrational frequency $\omega$, which satisfies the Diophantine condition 


$$
\|k \omega\|:=\operatorname{dist}(k \omega, \mathbb{Z}) \geq \frac{c}{|k|(\log |k|)^{2}}
$$

for some $c>0$ and for all $k \in \mathbb{Z} \backslash\{0\}$.

Moreover, in section 4.3 , we prove that almost every $\omega \in[0,1)$ satisfies the above Diophantine condition for some $c>0$ (with respect to the Lebesgue measure) (thus this condition is generic in a measure theoretical sense).

We say that a map $u: \mathbb{T} \rightarrow \mathbb{R}$ is $\alpha$-Hölder continuous if there exist nonnegative real constants $C$ and $\alpha$ such that

$$
|u(x)-u(y)| \leq C|x-y|^{\alpha}, \quad \forall x, y \in \mathbb{T} .
$$

We denote by $C^{\alpha}(\mathbb{T})$ the set of all $\alpha$-Hölder continuous functions on $\mathbb{T}$.

Given a continuous observable $u: \mathbb{T} \rightarrow \mathbb{R}$ and $n \in \mathbb{N}$, recall that

$$
S_{n} u(x):=\sum_{j=0}^{n-1} u\left(T^{j} x\right)=\sum_{j=0}^{n-1}(x+j \omega)
$$

are the corresponding Birkhoff sums. Moreover, recall that the Birkhoff averages $\frac{1}{n} S_{n} u(x)$ converge uniformly to the space average $\int_{\mathbb{T}} u$.

We estimate the convergence rate of the Birkhoff averages for Diophantine torus translation with a Hölder continuous observable. More precisely, we obtain the follow result.

Theorem 4.1.1 (S. Klein and A. Melo). Let $u: \mathbb{T} \rightarrow \mathbb{R}$ be an observable and let $\omega \in \mathbb{T}$ be an irrational frequency. Assume that $u$ is $\alpha$-Hölder continuous, for some $\alpha \in(0,1)$, and that $\omega$ satisfies the Diophantine condition (4.1).

There exists a universal constant $K$ such that for all $R \geq 1$ and $x \in \mathbb{T}$,

$$
\left|\frac{1}{R} \sum_{j=0}^{R-1} u(x+j \omega)-\int_{\mathbb{T}} u(x) d x\right| \leq \frac{K}{c} \frac{(\log R)^{3}}{R^{\alpha+1}}\|u\|_{\infty} .
$$

Results of this type are well know for observables with more regularity.

For example, given an observable $u \in C^{1+\alpha}(\mathbb{T})$ (meaning that $u$ is differentiable and $u^{\prime}$ is $\alpha$-Hölder), by means of solving the homological equation

$$
g(x+\omega)-g(x)=u(x)-\int_{\mathbb{T}} u,
$$

one can establish a rate of convergence of order $\frac{1}{R}$ for the Birkhoff sums (see sections 8.9 and 8.10 in [13]).

Moreover, the classical Denjoy-Koksma inequality provides a similar 
type of decay for observables of bounded variation, when choosing only the subsequence of Birkhoff sums corresponding to the denominators $q_{n}$ of the principal convergents of $\omega$ (see [14]).

The main ingredient of the proof of Theorem 4.1.1 is to combine the decay of the Fourier coefficients of the observable and the Diophantine condition (which helps control small denominators). The idea of the proof and the formal proof may be found in the section 4.4 and section 4.5 , respectively.

\section{2}

\section{Continued fractions}

Let $a_{0}, a_{1}, a_{2}, \ldots$ be independent variables. Define inductively pairs of polynomials

$$
p_{n}=p_{n}\left(a_{0}, \ldots, a_{n}\right) \text { and } q_{n}=q_{n}\left(a_{0}, \ldots, a_{n}\right)
$$

starting with $p_{0}=a_{0}$ and $q_{0}=1$. We denote the quotient $p_{n} / q_{n}$ by

$$
\frac{p_{n}}{q_{n}}=\left[a_{0}, \ldots, a_{n}\right]
$$

Suppose that we already defined $p_{k}, q_{k}$ with $k<n$, and $n \geq 1$. We use the notation

$$
p_{k}^{\prime}=p_{k}\left(a_{1}, \ldots, a_{n}\right) \text { and } q_{k}^{\prime}=q_{k}\left(a_{1}, \ldots, a_{n}\right) .
$$

Moreover, we define inductively

$$
p_{n}=a_{0} p_{n-1}^{\prime}+q_{n-1}^{\prime} \quad \text { and } \quad q_{n}=p_{n-1}^{\prime} .
$$

Then, by definition,

$$
\left[a_{0}, \ldots, a_{n}\right]=\frac{p_{n}}{q_{n}}=\frac{a_{0} p_{n-1}^{\prime}+q_{n-1}^{\prime}}{p_{n-1}^{\prime}}=a_{0}+\frac{1}{\frac{p_{n-1}^{\prime}}{q_{n-1}^{\prime}}}=a_{0}+\frac{1}{\frac{p_{n}\left(a_{1}, \ldots, a_{n}\right)}{q_{n}\left(a_{1}, \ldots, a_{n}\right)}}=a_{0}+\frac{1}{\left[a_{1}, \ldots, a_{n}\right]}
$$

which, written in full, is equal to

$$
a_{0}+\frac{1}{a_{1}+\frac{1}{a_{2}+\cdots}}
$$

The sequence of fractions $\left\{p_{n} / q_{n}\right\}$ is called a continued fraction. 
Theorem 4.2.1. For $n \geq 2$, we have that

$$
\begin{gathered}
p_{n}=a_{n} p_{n-1}+p_{n-2}, \\
q_{n}=a_{n} q_{n-1}+q_{n-2} .
\end{gathered}
$$

Proof. It is easy to see that the assertion is valid for $n=2$. Assume that the expression is valid for $n-1$ and, for $n>2$, assume inductively the following equalities

$$
\begin{aligned}
& p_{n-1}^{\prime}=a_{n} p_{n-2}^{\prime}+p_{n-3}^{\prime}, \\
& q_{n-1}^{\prime}=a_{n} q_{n-2}^{\prime}+q_{n-3}^{\prime} .
\end{aligned}
$$

Hence,

$$
\begin{aligned}
p_{n} & =a_{0} p_{n-1}^{\prime}+q_{n-1}^{\prime} \\
& =a_{0}\left(a_{n} p_{n-2}^{\prime}+p_{n-3}^{\prime}\right)+a_{n} q_{n-2}^{\prime}+q_{n-3}^{\prime} \\
& =a_{n}\left(a_{0} p_{n-2}^{\prime}+q_{n-2}^{\prime}\right)+a_{0} p_{n-3}^{\prime}+q_{n-3}^{\prime} \\
& =a_{n} p_{n-1}+p_{n-2} .
\end{aligned}
$$

Similarly, we have that

$$
q_{n}=a_{n} q_{n-1}+q_{n-2},
$$

which is the conclusion we were looking for.

Theorem 4.2.2. For $n \geq 0$, we have that

$$
q_{n} p_{n-1}-p_{n} q_{n-1}=(-1)^{n}
$$

Proof. We use induction in $n$. It is easy to see that for $n=0$ the above equality is satisfied. Thus, suppose that it is valid for $n$, that is,

$$
q_{n} p_{n-1}-p_{n} q_{n-1}=(-1)^{n}
$$

By Theorem 4.2.1, we have that

$$
\begin{aligned}
p_{n+1} & =a_{n+1} p_{n}+p_{n-1} \Rightarrow p_{n+1} q_{n}=a_{n+1} p_{n} q_{n}+p_{n-1} q_{n}, \\
q_{n+1} & =a_{n+1} q_{n}+q_{n-1} \Rightarrow q_{n+1} p_{n}=a_{n+1} q_{n} p_{n}+q_{n-1} p_{n} .
\end{aligned}
$$

Subtracting the first expression from the second and using the induction by, 
Chapter 4. A convergence rate for Birkhoff sums of Diophantine torus translations

we have that

$$
q_{n+1} p_{n}-p_{n+1} q_{n}=p_{n} q_{n-1}-q_{n} p_{n-1}=-\left(q_{n} p_{n-1}-p_{n} q_{n-1}\right)=(-1)^{n+1},
$$

which is the conclusion we were looking for.

Corollary 4.2.1. For $n \geq 1$, we have that

$$
\frac{p_{n-1}}{q_{n-1}}-\frac{p_{n}}{q_{n}}=\frac{(-1)^{n}}{q_{n} q_{n-1}}
$$

Proof. To obtain the above expression it is sufficient to divide the expression in Theorem 4.2 .2 by $q_{n} q_{n-1}$.

Corollary 4.2.2. If $a_{1}, a_{2}, \ldots$ are positive integers, then $p_{n}$ and $q_{n}$ are relative primes, and $0<q_{1}<q_{2}<\ldots$ forms a strictly increasing sequence of integers.

Proof. By Theorem 4.2.1,

$$
q_{n-1}<a_{n} q_{n-1} \leq a_{n} q_{n-1}+q_{n-2}=q_{n} \Rightarrow q_{n-1}<q_{n}, \forall n \in \mathbb{N}
$$

On the other hand, by Theorem 4.2.2,

$$
q_{n} p_{n-1}-p_{n} q_{n-2}=(-1)^{n}
$$

so $p_{n}$ and $q_{n}$ are relative prime.

Theorem 4.2.3. For $n \geq 1$, we have

$$
q_{n} p_{n-2}-p_{n} q_{n-2}=(-1)^{n-1} a_{n}
$$

Proof. By Theorem 4.2.1,

$$
\begin{gathered}
p_{n}=a_{n} p_{n-1}+p_{n-2} \Rightarrow p_{n} q_{n-2}=a_{n} p_{n-1} q_{n-2}+p_{n-2} q_{n-2}, \\
q_{n}=a_{n} q_{n-1}+q_{n-2} \Rightarrow q_{n} p_{n-2}=a_{n} q_{n-1} p_{n-2}+q_{n-2} p_{n-2} .
\end{gathered}
$$

Subtracting the first expression from the second and applying Theorem 4.2.2, we have that

$$
\begin{aligned}
q_{n} p_{n-2}-p_{n} q_{n-2} & =a_{n} q_{n-1} p_{n-2}-a_{n} p_{n-1} q_{n-2} \\
& =a_{n}\left(q_{n-1} p_{n-2}-p_{n-1} q_{n-2}\right) \\
& =(-1)^{n-1} a_{n},
\end{aligned}
$$

which is the conclusion we were looking for. 
Corollary 4.2.3. For $n \geq 2$, we have that

$$
\frac{p_{n-2}}{q_{n-2}}-\frac{p_{n}}{q_{n}}=\frac{(-1)^{n-1} a_{n}}{q_{n} q_{n-2}} .
$$

Proof. To obtain the above expression it is sufficient to divide the expression in Theorem 4.2 .3 by $q_{n} q_{n-2}$.

Corollary 4.2.4. If $a_{1}, a_{2}, \ldots$ are positive numbers, then, for even $n$, the sequence $p_{n} / q_{n}$ is strictly increasing and, for odd $n$, the sequence $p_{n} / q_{n}$ is strictly decreasing.

Proof. We claim that, for even $n$, the sequence is strictly increasing. Indeed, replace $n$ by $2 n$, then

$$
\begin{aligned}
q_{2 n} p_{2(n-1)}-p_{2 n} q_{2(n-1)}=(-1)^{2 n-1} a_{2 n} & \Rightarrow \frac{p_{2(n-1)}}{q_{2(k-1)}}-\frac{p_{2 n}}{q_{2 n}}=-\frac{a_{2 n}}{q_{2 n} q_{2(n-1)}} \\
& \Rightarrow \frac{p_{2(n-1)}}{q_{2(k-1)}}=\frac{p_{2 n}}{q_{2 n}}-\frac{a_{2 n}}{q_{2 n} q_{2(n-1)}}
\end{aligned}
$$

Hence, $\frac{p_{2(n-1)}}{q_{2(n-1)}}<\frac{p_{2 n}}{q_{2 n}}$, which is the conclusion we were looking for.

Similarly, we can prove that, for odd $n, p_{n} / q_{n}$ is a strictly decreasing sequence.

Corollary 4.2.5. Let $\alpha$ denote the rational number $\alpha=\left[a_{0}, \ldots, a_{n+2}\right]$. Then

$$
q_{n} \alpha-p_{n}=\frac{(-1)^{n} a_{n+2}}{a_{n+2} q_{n+1}+q_{n}} .
$$

Proof. By Theorem 4.2.3,

$$
q_{n+2} p_{n}-p_{n+2} q_{n}=(-1)^{n+1} a_{n+2} \Rightarrow p_{n}-\frac{p_{n+2}}{q_{n+2}} q_{n}=\frac{(-1)^{n+1} a_{n+2}}{q_{n+2}} .
$$

On the other hand, by Theorem 4.2.1, $q_{n+2}=a_{n+2} q_{n+1}+q_{n}$ and, by the definition, $\alpha=\frac{p_{n+2}}{q_{n+2}}$. Thus,

$$
q_{n} \alpha-p_{n}=\frac{(-1)^{n+2} a_{n+2}}{a_{n+2} q_{n+1}+q_{n}}=\frac{(-1)^{n} a_{n+2}}{a_{n+2} q_{n+1}+q_{n}} .
$$

Next we define and study the continued fraction of a real number. 
Let $\alpha$ be a rational number and let $a_{0}$ be the largest integer $\leq \alpha$. If $\alpha$ is not an integer then

$$
\alpha=a_{0}+\frac{1}{\alpha_{1}},
$$

where $\alpha_{1}>1$ is a rational number. Inductively, we let

$$
\alpha_{n}=a_{n}+\frac{1}{\alpha_{n+1}}
$$

with $a_{n}$ being the largest integer $\leq \alpha_{n}$ and $\alpha_{n+1}>1$. Observe that if $\alpha$ is a rational number, then the process will stop after a finite number of steps. Indeed, if $\alpha_{n}=a / b$ is a rational number such that $\alpha_{n} \notin \mathbb{Z}$. Then

$$
\alpha_{n}-a_{n}=\frac{a-b a_{n}}{b}=\frac{c}{b}
$$

Note that

$$
c=a-b a_{n}<b-b a_{n}=b \underbrace{\left(1-a_{n}\right)}_{<1}<b .
$$

Hence, $\alpha_{n+1}=\frac{b}{c}$ is a fraction whose its denominator is smaller than the denominator of $\alpha$. So, the process stops, and $\alpha$ can be written as

$$
\alpha=\left[a_{0}, \ldots, a_{n}\right]
$$

with $a_{0}, \ldots, a_{n} \in \mathbb{Z}$ and $a_{i} \geq 1$, for $i \geq 1$.

Now, consider $\alpha$ be an irrational number. As before, we can determine a continued fraction for $\alpha$, that is, we can write $\alpha=a_{0}+1 / \alpha_{1}$, with $a_{0}$ being the largest integer $\leq \alpha$. Inductively, we let

$$
\alpha_{n}=a_{n}+\frac{1}{\alpha_{n+1}},
$$

where $a_{n}$ is the largest integer $\leq \alpha_{n}$ and $\alpha_{n+1}>1$. Since $\alpha$ is irrational, the sequence $\left(a_{n}\right)$ does not end and we can represent $\alpha$ by

$$
\alpha=\left[a_{0}, a_{1}, \ldots\right],
$$

which we call its continued fraction expansion.

Thus, by Corollary 4.2.2, we obtain a sequence of relatively prime integers $p_{n}, q_{n}$ with $q_{n} \geq 1$. Let

$$
\frac{p_{n}}{q_{n}}=\left[a_{0}, \ldots, a_{n}\right] .
$$

Then, the fraction $p_{n} / q_{n}$ is called the $n$-th principal convergent of $\alpha$ and $a_{n}$ is 
called the $n$-th partial quotient of $\alpha$.

Theorem 4.2.4. For even $n$, the $n$-th principal convergents of $\alpha$ form a strictly increasing sequence converging to $\alpha$. On the other hand, for odd $n$, the $n$-th principal convergents of $\alpha$ form a strictly decreasing sequence converging to $\alpha$. Furthermore, we have

$$
\frac{1}{2 q_{n+1}}<\frac{1}{q_{n+1}+q_{n}}<\left|q_{n} \alpha-p_{n}\right|<\frac{1}{q_{n+1}}
$$

Proof. In Corollary 4.2.4, we proved the first part of the theorem. So, it is sufficient to prove the above inequalities.

a) $\frac{1}{2 q_{n+1}}<\frac{1}{q_{n+1}+q_{n}}$.

By Corollary 4.2.2,

$$
q_{n+1}+q_{n}<q_{n+1}+q_{n+1}=2 q_{n+1} \Rightarrow \frac{1}{2 q_{n+1}}<\frac{1}{q_{n+1}+q_{n}} .
$$

b) $\frac{1}{q_{n+1}+q_{n}}<\left|q_{n} \alpha-p_{n}\right|$.

First, note that

$$
a_{n+2} \geq 1 \Rightarrow \frac{1}{a_{n+2}} \leq 1 \Rightarrow q_{n+1}+\frac{q_{n}}{a_{n+2}} \leq q_{n+1}+q_{n} \Rightarrow \frac{1}{q_{n+1}+q_{n}} \leq \frac{1}{q_{n+1}+\frac{q_{n}}{a_{n+2}}}
$$

Therefore, applying Theorem 4.2.1 and Corollary 4.2.3, we have that

$$
\left|\alpha-\frac{p_{n}}{q_{n}}\right|>\left|\frac{p_{n+2}}{q_{n+2}}-\frac{p_{n}}{q_{n}}\right|=\frac{a_{n+2}}{q_{n+2} q_{n}}=\frac{a_{n+2}}{\left(a_{n+2} q_{n+1}+q_{n}\right) q_{n}}=\frac{1}{\left(q_{n+1}+\frac{q_{n}}{a_{n+2}}\right) q_{n}} .
$$

Hence,

$$
\left|q_{n} \alpha-p_{n}\right|>\frac{1}{q_{n+1}+\frac{q_{n}}{a_{n+2}}} \leq \frac{1}{q_{n+1}+q_{n}} .
$$

c) $\left|q_{n} \alpha-p_{n}\right|<\frac{1}{q_{n+1}}$

Since $q_{n+1}<q_{n+1}+\frac{q_{n}}{a_{n+2}}$, by Corollary 4.2.5, we have that

$$
\left|q_{n} \alpha-p_{n}\right|=\frac{a_{n+2}}{q_{n+1}+a_{n+2} q_{n}}=\frac{1}{q_{n+1}+\frac{q_{n}}{a_{n+2}}}<\frac{1}{q_{n+1}} .
$$

The following picture illustrate the Theorem 4.2.4 
Chapter 4. A convergence rate for Birkhoff sums of Diophantine torus translations

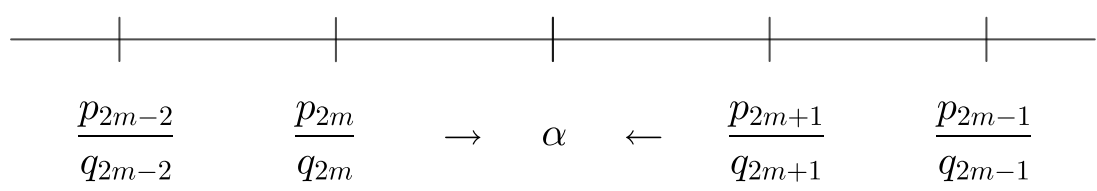

Figure 4.1: Theorem 4.2.4 illustration

\section{3}

\section{The Diophantine condition}

Let $\alpha$ be a real number. We denote by $\|\alpha\|$ the distance between $\alpha$ and the nearest integer.

Definition 4.3.1. Let $\alpha$ be a real number. We say that a fraction $\frac{p}{q},(q>0)$, is a best approximation to $\alpha$ if

$$
\|q \alpha\|=|q \alpha-p| \quad \text { and } \quad\left\|q^{\prime} \alpha\right\|>\|q \alpha\|
$$

for every $1 \leq q^{\prime}<q$.

Theorem 4.3.1. The best approximations to $\alpha$ are the principal convergents. In fact, $q_{n+1}$ is the smallest integer $q^{\prime}>q_{n}$ such that $\left\|q^{\prime} \alpha\right\|<\left\|q_{n} \alpha\right\|$.

Proof. Le $a / b$ be a reduced fraction, which is a best approximation to $\alpha$, where $b>0$. We claim that $a / b$ is a principal convergent, that is, $a / b=p_{n} / q_{n}$, for some $n \in \mathbb{N}$.

First, suppose that $a / b<p_{0} / q_{0}=a_{0}$. Since $q_{0}=1$, we have that

$$
\left|q_{0} \alpha-p_{0}\right|=\left|\alpha-\frac{p_{0}}{q_{0}}\right|=\left|\alpha-a_{0}\right|<\left|\alpha-\frac{a}{b}\right|=\left|\frac{b \alpha-a}{b}\right| \leq|b \alpha-a| .
$$

Thus, $\left\|q_{0} \alpha\right\|=\left|q_{0} \alpha-p_{0}\right|<|b \alpha-a|=\|b \alpha\|$, contradicting the hypothesis because $q_{0}<b$. Suppose, now, that $a / b>p_{1} / q_{1}$. Thus,

$$
\frac{a}{b}>\frac{p_{1}}{q_{1}} \Rightarrow a q_{1}>b p_{1} \Rightarrow a q_{1}-b p_{1}>0 \Rightarrow a q_{1}-b p_{1} \geq 1
$$

because $a, b, p_{1}, q_{1} \in \mathbb{Z}$, so $a q_{1}-b p_{1} \in \mathbb{Z}$. But, by Theorem $4.2 .4, \alpha<p_{1} / q_{1}$, then,

$$
\left|\frac{a}{b}-\alpha\right|>\left|\frac{a}{b}-\frac{p_{1}}{q_{1}}\right| \geq \frac{1}{b q_{1}} \Rightarrow|b \alpha-a|>\frac{1}{q_{1}} .
$$

Hence, by Theorem 4.2.4,

$$
\left|\alpha-a_{0}\right|=\left|\alpha-\frac{p_{0}}{q_{0}}\right|=\left|\frac{q_{0} \alpha-p_{0}}{q_{0}}\right|<\frac{1}{q_{0} q_{1}}=\frac{1}{q_{1}}=\frac{1}{a_{1}} .
$$


Then

$$
|b \alpha-a|>\frac{1}{q_{1}}=\frac{1}{a_{1}} \geq\left|\alpha-a_{0}\right|
$$

again contradicting the hypothesis. Now, suppose that $a / b$ is such that $p_{n-1} / q_{n-1}<a / b<p_{n+1} / q_{n+1}$. Then

$$
\frac{1}{b q_{n-1}} \leq\left|\frac{a}{b}-\frac{p_{n-1}}{q_{n-1}}\right|<\left|\frac{p_{n}}{q_{n}}-\frac{p_{n-1}}{q_{n-1}}\right|=\frac{1}{q_{n} q_{n-1}}
$$

by Theorem 4.2.2. Therefore, $q_{n}<b$. But,

$$
\frac{1}{b q_{n+1}} \leq\left|\frac{p_{n+1}}{q_{n+1}}-\frac{a}{b}\right| \leq\left|\alpha-\frac{a}{b}\right| \text {. }
$$

Again, by Theorem 4.2.4, we have that

$$
\left\|q_{n} \alpha\right\|=\left|q_{n} \alpha-p_{n}\right|<\frac{1}{q_{n+1}} \leq b\left|\alpha-\frac{a}{b}\right|=|b \alpha-a| \Rightarrow\left\|q_{n} \alpha\right\|<\|b \alpha\|, q_{n}<b
$$

contradicting the hypothesis. Thus, there exists $n \in \mathbb{N}$ such that $a / b=p_{n} / q_{n}$.

The second half of the Theorem, is proven by induction on $n$. It is obvious for $n=0$, because $q_{0}=1$ and there is no $q \in \mathbb{N}$ such that $1 \leq q<q_{0}$. Hence, $p_{0} / q_{0}$ satisfies the the definition of best approximation by vacuity.

Assume that our assertion is valid for $p_{n} / q_{n}$, with $n \geq 0$. We will show that $p_{n+1} / q_{n+1}$ is a best approximation. Let $q$ be the smallest integer $>q_{n}$ such that $\|q \alpha\|<\left\|q_{n} \alpha\right\|$, and let $p$ be such that $\|q \alpha\|=|q \alpha-p|$. Then, by the inductive property, we conclude that $p / q$ is a best approximation to $\alpha$. By the first part of the Theorem, $p / q=p_{n} / q_{n}$, for some $n \in \mathbb{N}$. Since $q$ is the smallest integer $>q$ such that $\|q \alpha\|<\left\|q_{n} \alpha\right\|$, we conclude that $q=q_{n+1}$. Thus, $p=p_{n+1}$, thereby proving our theorem.

Corollary 4.3.1. If $\frac{p}{q}$ is a principal convergent to $\alpha$ and $m \in \mathbb{Z}$ is such that $1 \leq m<q$, then $\|m \alpha\|>\frac{1}{2 q}$.

Proof. Suppose that $q=q_{n}$. By Theorem 4.2.4,

$$
\frac{1}{2 q_{n}}<\left|q_{n} \alpha-p_{n}\right|=\left\|q_{n-1} \alpha\right\| .
$$

Since $m<q_{n}$ and applying Theorem 4.3.1, we have that $\left\|q_{n-1} \alpha\right\|<\|m \alpha\|$.

Definition 4.3.2. We say that an irrational number $\alpha$ satisfies a Diophantine condition if for some $c \in \mathbb{R}, c>0$ and for all $k \in \mathbb{Z} \backslash\{0\}$, 


$$
\|k \alpha\|:=\operatorname{dist}(k \alpha, \mathbb{Z}) \geq \frac{c}{|k|(\log |k|)^{2}} .
$$

Theorem 4.3.2. Almost every number $\alpha \in[0,1]$ satisfies a Diophantine condition for some $c>0$. In other words, for almost every $\alpha \in[0,1)$ there is only a finite number of solutions $k \in \mathbb{Z}$ to the inequality $\|k \alpha\| \leq \frac{1}{|k|(\log |k|)^{2}}$.

Proof. Define $\psi(q)=\frac{1}{|q|(\log |q|)^{2}}$. Let

$$
B=\{\alpha \in[0,1]:\|q \alpha\|<\psi(q) \text { has infinitely many solution }\}
$$

We have to show that $\lambda(B)=0$, where $\lambda$ denotes the Lebesgue measure.

Given $\epsilon>0$, take $q_{0} \in \mathbb{N}$ such that the $\sum_{q \geq q_{0}}^{\infty} \psi(q)<\epsilon$. This is possible since the series converge.

For every $q \geq q_{0}$, consider the intervals

$$
\begin{aligned}
I_{0} & =\left(-\frac{\psi(q)}{q}, \frac{\psi(q)}{q}\right) \\
I_{1} & =\left(\frac{1}{q}-\frac{\psi(q)}{q}, \frac{1}{q}+\frac{\psi(q)}{q}\right) \\
\vdots & \\
I_{q-1} & =\left(\frac{q-1}{q}-\frac{\psi(q)}{q}, \frac{q-1}{q}+\frac{\psi(q)}{q}\right) .
\end{aligned}
$$

If $\alpha \in B$ then $\alpha \in I_{j}$, for some $j \in\{0, \ldots, q-1\}$ since $\|q \alpha\|<\psi(q)$ and

$$
\left|\alpha-\frac{p}{q}\right|<\frac{\psi(q)}{q}
$$

Since $B \subset \bigcup_{k=0}^{q-1} I_{k}$, we have that

$$
\lambda(B) \leq \lambda\left(\bigcup_{k=0}^{q-1} I_{k}\right)=\sum_{q \leq q_{0}} q \frac{2 \psi(q)}{q}<2 \epsilon
$$

which is the conclusion we were looking for.

Lemma 4.3.3. Assume that $\omega$ be an irrational number which satisfies the Diophantine condition (4.2) Then, for every large enough integer $R$, there 
exists a best approximation $\frac{p}{q}$ to $\omega$ such that

$$
R<q<\frac{R(\log R)^{2}}{c}
$$

Moreover, if $1 \leq j<q$ then $\|j \omega\|>\frac{1}{2 q}$.

Proof. Let $\omega$ be a irrational number which satisfies the Diophantine condition and consider its continued fraction representation $\omega=\left[a_{0}, a_{1}, \ldots, a_{n}, \ldots\right]$. For every $n \geq 1$, let

$$
\frac{p_{n}}{q_{n}}=\left[a_{0}, a_{1}, \ldots, a_{n}\right]
$$

be its $n$-th principal convergent. By Theorem 4.2.4,

$$
\frac{1}{2 q_{n+1}}<\left\|q_{n} \omega\right\|=\left|q_{n} \omega-p_{n}\right|<\frac{1}{q_{n+1}} .
$$

Then, for any $R \in \mathbb{N}$ sufficiently large, let $n+1$ be the first integer $j$ such that $q_{j}>R$. Then, $q_{n} \leq R<q_{n+1}$, and using the inequalities (4.3) and (4.2), we have that

$$
q_{n+1}<\frac{1}{\left\|q_{n} \omega\right\|} \leq \frac{q_{n}\left(\log q_{n}\right)^{2}}{c}<\frac{R(\log R)^{2}}{c} .
$$

Let the fraction $\frac{p}{q}$ be a best approximation to $\omega$, that is, $\|q \omega\|=|q \omega-p|$ and

$$
\|j \omega\|>\|q \omega\| \text { for all } 1 \leq j<q .
$$

By Theorem 4.3.1, $q=q_{n+1}$ for some $n \geq 0$, thereby

$$
R<q<\frac{R(\log R)^{2}}{c}
$$

Moreover, for every $1 \leq j<q=q_{n+1}$, we have that $\|j \omega\| \geq\left\|q_{n} \omega\right\|$. It follows from the inequality (4.3) that

$$
\left\|q_{n} \omega\right\|>\frac{1}{2 q_{n+1}}=\frac{1}{2 q}
$$

Hence, if $\frac{p}{q}$ is a best approximation to $\omega$, we have that

$$
\|j \omega\|>\frac{1}{2 q} \quad \text { for all } \quad 1 \leq j<q
$$

which is the conclusion we were looking for. 
Chapter 4. A convergence rate for Birkhoff sums of Diophantine torus translations

\section{4}

\section{Convergence rate of Birkhoff sums: idea of the proof}

In this section we give an intuitive idea of the proof of Theorem 4.1.1.

Recall that a function $u: \mathbb{T} \rightarrow \mathbb{R}$ is $\alpha$-Hölder continuous if there exist non-negative real constants $C$ and $\alpha$ such that

$$
|u(x)-u(y)| \leq C|x-y|^{\alpha}, \quad \forall x, y \in \mathbb{T} .
$$

We denote by $C^{\alpha}(\mathbb{T})$ the set of all $\alpha$-Hölder continuous functions on $\mathbb{T}$.

Given a function $u \in C^{\alpha}(\mathbb{T})$, its Fourier series

$$
u(x)=\sum_{k \in \mathbb{Z}} \hat{u}(k) e^{2 \pi i k x}
$$

converges for every $x \in \mathbb{T}$, where

$$
\hat{u}(k)=\int_{\mathbb{T}} u(x) e^{-2 \pi i k x} d x
$$

is the $k$-th Fourier coefficient of $u$.

Then

$$
\hat{u}(0)=\int_{\mathbb{T}} u(x) d x
$$

Hence

$$
u(x)=\int_{\mathbb{T}} u+\sum_{k \neq 0} \hat{u}(k) e^{2 \pi i k x} .
$$

Since

$$
\left(S_{R} u\right)(x)=u(x)+u(x+\omega)+\cdots+u(x+(R-1) \omega),
$$

we have that

$$
\frac{1}{R}\left(S_{R} u\right)(x)=\int_{\mathbb{T}} u+\sum_{k \neq 0} \hat{u}(k) \frac{1}{R} S_{R}\left(e^{2 \pi i k x}\right) .
$$

A simple calculation shows that

$$
\frac{1}{R} S_{R}\left(e^{2 \pi i k x}\right)=e^{2 \pi i k x} \frac{1}{R} \sum_{j=0}^{R-1} e^{2 \pi i j k \omega}
$$

Denoting

$$
F_{R}(k \omega):=\frac{1}{R} \frac{e^{2 \pi i R k \omega}-1}{e^{2 \pi i k \omega}-1}
$$


and substituting the above in (4.4), we obtain

$$
\frac{1}{R}\left(S_{R} u\right)(x)-\int_{\mathbb{T}} u=\sum_{k \neq 0} \hat{u}(k) F_{R}(k \omega) e^{2 \pi i k x} .
$$

Thus

$$
\left|\frac{1}{R}\left(S_{R} u\right)(x)-\int_{\mathbb{T}} u\right| \leq \sum_{k \neq 0}|\hat{u}(k)|\left|F_{R}(k \omega)\right|
$$

uniformly in $x \in \mathbb{T}$.

Hence we have to stimate the right-hand side on the previous inequality. Note that

$$
\left|F_{R}(k \omega)\right| \leq \min \left\{1, \frac{1}{R} \frac{1}{\|k \omega\|}\right\}
$$

Since $\omega$ satisfies the Diophantine condition (4.1), we have that

$$
\frac{1}{\|k \omega\|} \leq \frac{|k|(\log |k|)^{2}}{c}
$$

Then

$$
\left|F_{R}(k \omega)\right| \leq \min \left\{1, \frac{1}{R} \frac{1}{\|k \omega\|}\right\} \leq \frac{1}{R} \cdot \frac{|k|(\log |k|)^{2}}{c}
$$

On the other hand, since $u \in C^{\alpha}(\mathbb{T})$, its Fourier coefficients have the decay

$$
|\hat{u}(k)| \leq \frac{C}{|k|^{\alpha}} .
$$

These two pieces of information alone, the decay of the kernel $F_{R}(k \omega)$ (provided by the Diophantine condition) and the decay of the Fourier coefficients $\hat{u}(k)$ (provided by the Hölder regularity of the observable), while helpful, are not enough to establish the desired uniform estimate. That is because of the following two issues.

1) The right hand side of (4.5) is an infinite sum, so when $k$ is of order $R$ (or higher), the upper bound (4.6), as written, will stop being useful.

2) When the order $\alpha$ of the Hölder class is very close to zero, the decay rate (4.7) becomes too weak.

To overcome these issues, we use approximations of the observable $u$ by trigonometric polynomials, whose Fourier series expansions are finite. 
By Jackson's Theorem, there exists a universal constant $C<\infty$ and for all $n \geq 1$, there exists a trigonometric polynomial $p_{n}$ of degree $n$ such that

$$
\left\|u-p_{n}\right\|_{\infty} \leq C n^{-\alpha}
$$

Let $p$ be such a trigonometric polynomial of degree $n$, so

$$
p(x)=\sum_{k=-n}^{n} \hat{p}(k) e^{2 \pi i k x} .
$$

The calculation done above for $u$, applied to $p$ yields

$$
\left|\frac{1}{R}\left(S_{R} p\right)(x)-\int_{\mathbb{T}} p\right| \leq \sum_{0<|k| \leq n}|\hat{p}(k)|\left|F_{R}(k \omega)\right| .
$$

where again

$$
\left|F_{R}(k \omega)\right| \leq \min \left\{1, \frac{1}{R} \cdot \frac{1}{\|k \omega\|}\right\} \lesssim \frac{|k|(\log |k|)^{2}}{R}
$$

Moreover, we have the stronger Fourier coefficients bound

$$
|\hat{p}(k)| \leq \frac{C}{|k|}
$$

where the constant $C$ is of order $n\|p\|_{\infty}$.

The key idea of the proof is to choose, for every $R$ (the length of the Birkhoff sum) an appropriate $n$ (degree of polynomial approximation) and to work with the corresponding trigonometric polynomial $p_{n}$ instead of the original observable $u$. In other words, for every iterate $R$, we choose a different observable $p_{n} \in \mathcal{T}_{n}$ (where $n$ is related to $R$ ) which approximates $u$ well (so the resulting error is small) and avoids the issues mentioned above. The relevant estimate for the approximant $p_{n}$ is formalized in the technical result below.

Lemma 4.4.1. Let $p \in \mathcal{T}_{n}$ and let $\omega \in \mathbb{T}$. Assume that $\omega$ satisfies the Diophantine condition (4.2) and let $R \in \mathbb{N}$ be such that $\log R<n \leq R$. Then

$$
\left\|\frac{1}{R} S_{R} p-\int_{\mathbb{T}} p\right\|_{\infty} \leq \frac{12}{c} \frac{(\log R)^{3}}{\pi R} n\|p\|_{\infty}
$$

The idea of the proof of Lemma 4.4.1 is to use approximations of $\omega$ by continued fractions. 
In Section 4.3, we prove that for large enough integers $R$, there exists a best approximation $\frac{p}{q}$ to $\omega$ such that

$$
q>\log \mathbb{R} \quad \text { but } q \approx \log R
$$

Hence, we split the right hand side of the sum in the equality (4.8) as:

$$
\sum_{0<|k| \leq n}|\hat{p}(k)|\left|F_{R}(k \omega)\right|=\sum_{0<|k|<\log R}+\sum_{\log R \leq|k|<q}+\sum_{q \leq|k| \leq n}
$$

Moreover, in Section 4.5, we prove that for any interval $I \subset \mathbb{Z}$ of size $\leq q$, we have that

$$
\sum_{k \in I}\left|F_{R}(k \omega)\right| \lesssim \frac{8 q}{R} \log R
$$

Thus, in order to obtain a sharp upper bound, we divide the last sum in (4.9) into sums of sizes $\leq q$,

$$
\sum_{q \leq|k| \leq n}=\sum_{1 \leq s \leq \frac{n}{q}} \sum_{s q \leq|k|<(s+1) q}
$$

which are estimated individually using (4.10).

The following picture illustrates the division into the three sums in (4.9).

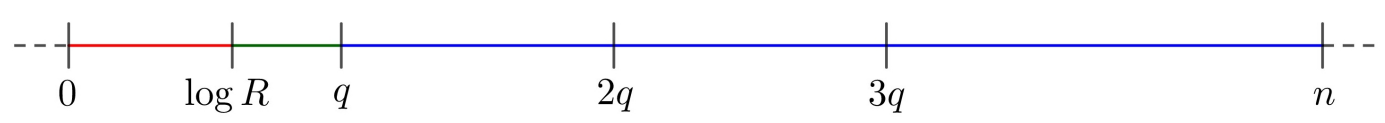

Figure 4.2: Illustration of the proof

\section{5}

\section{Convergence rate of Birkhoff sums: formal proof}

In this section we establish Theorem 4.1.1 on the convergence rate for the Birkhoff sums of a Hölder observable, over a Diophantine torus translation. Our approach follows ideas used in the study of quasi-periodic linear cocycles, see for instance [15]. To the best of our knowledge, this result is new.

We recall that a function $u: \mathbb{T} \rightarrow \mathbb{R}$ is said to be $\alpha$-Hölder continuous if there exist non-negative real constants $C$ and $\alpha$ such that

$$
|u(x)-u(y)| \leq C|x-y|^{\alpha}, \quad \forall x, y \in \mathbb{T} .
$$

We denote by $C^{\alpha}(\mathbb{T})$ the set of all $\alpha$-Hölder continuous functions on $\mathbb{T}$. 
Our approach uses approximations of the observable by trigonometric polynomials. We recall bellow two important results that will be crucial in our proof (see p.49 in [13])

Theorem 4.5.1 (Jackson). If $u \in C^{\alpha}(\mathbb{T})$ then, there exists a universal constant $C<\infty$ and for all $n \geq 1$, there exists $p_{n}$ trigonometric polynomial of degree $n$ such that

$$
\left\|u-p_{n}\right\|_{\infty} \leq C n^{-\alpha}
$$

Theorem 4.5.2 (Bernstein). If $p_{n}$ is a trigonometric polynomial of degree $n$, then

$$
\left\|p_{n}^{\prime}\right\|_{\infty} \leq n\left\|p_{n}\right\|_{\infty}
$$

The key ingredient in our proof is combining the decay of the Fourier coefficients of the observable and the Diophantine condition.

We recall that given a function $u \in L^{2}(\mathbb{T})$, its $k$-th Fourier coefficient is

$$
\widehat{u}(k)=\int_{0}^{1} u(x) e(-k x) d x
$$

where $e(x)=e^{2 \pi i x}$.

We recall that $\mathcal{T}_{n}$ is the set of all trigonometric polynomials of degree $\leq n$. In other words, if $p \in \mathcal{T}_{n}$, then

$$
p(x)=\sum_{k=-n}^{n} a_{k} e(k x)
$$

where $a_{k} \in \mathbb{C}$ with $k=-1, \ldots, n$. It is easy to verify that in fact $a_{k}=\widehat{p}(k)$ for all $k$, where $\hat{p}(k)$ is the $k$-th Fourier coefficient of $p$.

Lemma 4.5.3. Let $p \in \mathcal{T}_{n}$ and let $\hat{p}(k)$ be the $k$-th Fourier coefficient of $p$. Then,

$$
|\hat{p}(k)| \leq \frac{n\|p\|_{\infty}}{2 \pi|k|}
$$

Proof. Let $k \in \mathbb{Z}$. Since $p \in \mathcal{T}_{n}$, we can write $p$ as

$$
p(x)=\sum_{k=-n}^{n} \hat{p}(k) e(k x) .
$$

Then, by the definition of the Fourier coefficients and using integration 
by parts, we have that

$$
\begin{aligned}
\hat{p}(k) & =\int_{0}^{1} p(x) \overline{e(k x)} d x=\int_{0}^{1} p(x) e(-k x) d x \\
& =\underbrace{\left.-\frac{1}{2 \pi i k} p(x) e(-k x)\right]_{0}^{1}}_{=0}-\frac{1}{2 \pi i k} \int_{0}^{1} p^{\prime}(x) e(-k x) d x \\
& =-\frac{1}{2 \pi i k} \int_{0}^{1} p^{\prime}(x) e(-k x) d x .
\end{aligned}
$$

Hence, by Theorem 4.5.2,

$$
|\hat{p}(k)|=\left|-\frac{1}{2 \pi i k} \int_{0}^{1} p^{\prime}(x) e(-k x) d x\right| \leq \frac{1}{2 \pi i k} \int_{0}^{1}\left|p^{\prime}(x) e(-k x)\right| d x \leq \frac{n\|p\|_{\infty}}{2 \pi|k|} .
$$

Lemma 4.5.4. Let $p \in \mathcal{T}_{n}$ and let $\omega \in \mathbb{T}$. Assume that $\omega$ satisfies the Diophantine condition (4.2) and let $R \in \mathbb{N}$ be such that $\log R<n \leq R$. Consider the translation map $T_{\omega}$, where $T_{\omega}(x)=x+\omega$. Then,

$$
\left\|\frac{1}{R} S_{R} p-\int p\right\|_{\infty} \leq \frac{12}{c} \frac{(\log R)^{3}}{\pi R} n\|p\|_{\infty} .
$$

where $\left(S_{R} p\right)(x)=p(x)+p\left(T_{\omega} x\right)+\cdots+p\left(T_{\omega}^{R-1} x\right)$.

Proof. Since $p$ is a trigonometric polynomial of degree $n$, we have that

$$
\begin{aligned}
p(x) & =\sum_{k=-n}^{n} \hat{p}(k) e(k x)=\widehat{p}(0)+\sum_{0<|k| \leq n} \hat{p}(k) e(k x) \\
& =\int p+\sum_{0<|k| \leq n} \hat{p}(k) e(k x) .
\end{aligned}
$$

Then, for $j \in \mathbb{Z}$,

$$
p(x+j \omega)-\int p=\sum_{0<|k| \leq n} \hat{p}(k) e(k(x+j \omega))=\sum_{0<|k| \leq n} \hat{p}(k) e(j k \omega) e(k x) .
$$


Therefore,

$$
\begin{aligned}
\frac{1}{R}\left(S_{R} p\right)(x)-\int p & =\frac{1}{R} \sum_{j=0}^{R-1} p(x+j \omega)-\int p \\
& =\frac{1}{R} \sum_{j=0}^{R-1}\left(p(x+j \omega)-\int p\right) \\
& =\frac{1}{R} \sum_{j=0}^{R-1}\left(\sum_{0<|k| \leq n} \hat{p}(k) e(j k \omega) e(k x)\right) \\
& =\sum_{0<|k| \leq n} \hat{p}(k)\left(\frac{1}{R} \sum_{j=0}^{R-1} e(j k \omega)\right) e(k x) .
\end{aligned}
$$

Consider the Féjer-type kernel of order 1:

$$
F_{R}(t):=\frac{1}{R} \sum_{j=0}^{R-1} e(j t)
$$

It follows that

$$
\frac{1}{R} \sum_{j=0}^{R-1} p(x+j \omega)-\int p=\sum_{0<|k| \leq n} \hat{p}(k) F_{R}(k \omega) e(k x) .
$$

Hence, it is enough to estimate the right hand side of the equality (4.11). By Lemma 4.5.3,

$$
|\hat{p}(k)| \leq \frac{n\|p\|_{\infty}}{2 \pi|k|}
$$

We also have to estimate $F_{R}(k \omega)$. Since $F_{R}(k \omega)$ is the sum of a finite geometric sequence, it follows that

$$
\left|F_{R}(t)\right|=\frac{1}{R}\left|\frac{e(t)^{R}-1}{e(t)-1}\right|=\frac{1}{R}\left|\frac{1-e(R t)}{1-e(t)}\right| \leq \frac{1}{R\|t\|} .
$$

On the other hand, $\left|F_{R}(t)\right| \leq 1$, then the kernel $F_{R}(t)$ satisfies the bound

$$
\left|F_{R}(t)\right| \leq \min \left\{1, \frac{1}{R\|t\|}\right\}
$$

Hence, we have two cases:

- $\min \left\{1, \frac{1}{R\|t\|}\right\}=1 \Rightarrow 1 \leq \frac{1}{R\|t\|} \Rightarrow R\|t\| \leq 1 \Rightarrow\left|F_{R}(t)\right| \leq 1 \leq \frac{2}{1+R\|t\|}$

- $\min \left\{1, \frac{1}{R\|t\|}\right\}=\frac{1}{R\|t\|} \Rightarrow \frac{1}{R\|t\|} \leq 1 \Rightarrow 1+R\|t\| \leq 2 R\|t\| \Rightarrow \frac{1}{R\|t\|} \leq \frac{2}{1+R\|t\|}$

Therefore, 


$$
\left|F_{R}(t)\right| \leq \min \left\{1, \frac{1}{R\|t\|}\right\} \leq \frac{2}{1+R\|t\|}
$$

Since $\omega$ satisfies the Diophantine condition, it follows from Lemma 4.3.3 applied to the integer $\lfloor\log R\rfloor$ that there exists a best approximation $\frac{p}{q}$ to $\omega$ such that

$$
\log R<q<\frac{\log R[\log (\log R)]^{2}}{c}
$$

Moreover,

$$
\|j \omega\|>\frac{j}{2 q}, \quad 1 \leq j<q
$$

Split the right hand side of the sum in the equality (4.11) as:

$$
\sum_{0<|k| \leq n} \hat{p}(k) F_{R}(k \omega) e(k x)=\sum_{0<|k|<\log R}+\sum_{\log R \leq|k|<q}+\sum_{q \leq|k| \leq n}
$$

Denote by $S_{1}(x), S_{2}(x)$ and $S_{3}(x)$ the three sums on the right side of the equality (4.16). So, we will each of estimate these three sums separately.

From (4.12) and (4.13), we get

$$
\left|S_{1}(x)\right| \leq \sum_{0<|k|<\log R}|\hat{p}(k)|\left|F_{R}(k \omega)\right| \leq \frac{n\|p\|_{\infty}}{2 \pi R} \sum_{0<|k|<\log R} \frac{1}{|k|\|k \omega\|} .
$$

Using the Diophantine condition (4.2), we have that

$$
\frac{c}{|k|(\log |k|)^{2}} \leq\|k \omega\| \Rightarrow \frac{1}{|k|\|k \omega\|} \leq \frac{(\log |k|)^{2}}{c}
$$

Then, for every $x \in \mathbb{T}$,

$$
\begin{aligned}
\left|S_{1}(x)\right| & \leq \frac{n\|p\|_{\infty}}{2 \pi R} \sum_{0<|k|<\log R} \frac{1}{|K|\|k \omega\|} \leq \frac{n\|p\|_{\infty}}{2 \pi R c} \sum_{0<|k|<\log R}(\log |k|)^{2} \\
& \leq \frac{n\|p\|_{\infty}}{2 \pi R c} \sum_{0<|k|<\log R}[\log (\log R)]^{2} \\
& \leq \frac{n\|p\|_{\infty}}{2 \pi R c} \cdot \log R \cdot[\log (\log R)]^{2} .
\end{aligned}
$$

To bound $S_{2}(x)$ and $S_{3}(x)$ we need the following estimate.

Let $I \subset \mathbb{Z}$ be any interval of size $|I|<q$. Then, for every $k, k^{\prime} \in I$ with $k \neq k^{\prime}$, we have that $\left|k-k^{\prime}\right| \leq|I|<q$. Hence, the inequality (4.15) implies

$$
\left\|k \omega-k^{\prime} \omega\right\|=\left\|\left(k-k^{\prime}\right) \omega\right\|>\frac{1}{2 q} .
$$


Divide $\mathbb{T}$ into the $2 q$ arcs

$$
C_{j}=\left[\frac{j}{2 q}, \frac{j+1}{2 q}\right), \quad 0 \leq j \leq 2 q-1
$$

with size $\left|C_{j}\right|=\frac{1}{2 q}$. By the observation in (4.17), each arc $C_{j}$ contains at most one point $k \omega$, with $k \in I$.

Moreover, if $x \in C_{j}$, with $0 \leq j \leq q-1$, then $x \in\left[0, \frac{1}{2}\right)$ and, consequently, $\|x\| \geq \frac{j}{2 q}$. On the other hand, if $x \in C_{k}$, with $q \leq k \leq 2 q-1$, thus $x \in\left[\frac{1}{2}, 1\right)$. In this case, $\|x\| \geq 1-\frac{k+1}{2 q}=\frac{2 q-k+1}{2 q}$. Take $j=2 q-k-1$, then $k=2 q-j-1$ and

$$
q \leq k \leq 2 q-1 \Rightarrow 0 \leq 2 q-k-1 \leq q-1 \Rightarrow 0 \leq j \leq q-1 .
$$

Hence, if $x \in C_{k}$ with $q \leq k \leq 2 q-1$, it follows that $x \in C_{2 q-j-1}$ with $0 \leq j \leq q-1$. On the other hand, note that

$$
\|k \omega\| \geq \frac{j}{2 q} \Rightarrow 1+R\|k \omega\| \geq 1+R\left(\frac{j}{2 q}\right) \Rightarrow \frac{1}{1+R\|k \omega\|} \leq \frac{1}{1+R\left(\frac{j}{2 q}\right)}
$$

for any $k \omega \in C_{j}$ and $k \omega \in C_{2 q-j-1}$, with $0 \leq j \leq q-1$.

On the other hand,

- $R>2 \Leftrightarrow 1+\frac{R}{2}<R \Leftrightarrow \log \left(1+\frac{R}{2}\right)<\log R \Leftrightarrow \frac{8 q}{R} \log \left(1+\frac{R}{2}\right)<\frac{8 q}{R} \log R$.

Then, by the observations above and the inequality (4.13), we have that

$$
\begin{aligned}
\sum_{k \in I}\left|F_{R}(k \omega)\right| & \leq \sum_{k \in I} \frac{2}{1+R\|k \omega\|} \leq \sum_{j=0}^{q-1} \frac{4}{1+R\left(\frac{j}{2 q}\right)} \\
& \leq \int_{0}^{q} \frac{4}{1+R\left(\frac{x}{2 q}\right)} d x \\
& =\frac{q}{R}\left[8 \log \left(1+R\left(\frac{x}{2 q}\right)\right)\right]_{0}^{q} \\
& =\frac{q}{R}\left[8 \log \left(1+\frac{R}{2}\right)\right] \\
& \lesssim \frac{8 q}{R} \log R .
\end{aligned}
$$

Hence, for any interval $I \subset \mathbb{Z}$ of size $<q$,

$$
\sum_{k \in I}\left|F_{R}(k \omega)\right| \lesssim \frac{8 q}{R} \log R
$$


Using the inequalities (4.18) and (4.14), it follows that for every $x \in \mathbb{T}$,

$$
\begin{aligned}
\left|S_{2}(x)\right| & \leq \sum_{\log R \leq|k|<q}|\hat{p}(k)|\left|F_{R}(k \omega)\right| \\
& \leq \frac{n\|p\|_{\infty}}{2 \pi} \sum_{\log R \leq|k|<q} \frac{1}{|k|}\left|F_{R}(k \omega)\right| \\
& \leq \frac{n\|p\|_{\infty}}{2 \pi \log R} \sum_{1 \leq|k|<q}\left|F_{R}(k \omega)\right| \\
& \leq \frac{n\|p\|_{\infty}}{2 \pi \log R} \cdot \frac{8 q}{R} \log R \leq \frac{4 n\|p\|_{\infty}}{\pi R} \cdot \frac{\log R \cdot[\log (\log R)]^{2}}{c} .
\end{aligned}
$$

Similarly,

$$
\begin{aligned}
\left|S_{3}(x)\right| & \leq \sum_{q \leq|k|<n}|\hat{p}(k)|\left|F_{R}(k \omega)\right| \leq \frac{n\|p\|_{\infty}}{2 \pi} \sum_{q \leq|k|<n} \frac{1}{|k|}\left|F_{R}(k \omega)\right| \\
& \leq \frac{n\|p\|_{\infty}}{2 \pi} \sum_{1 \leq s \leq \frac{n}{q}}\left(\sum_{s q \leq|k|<(s+1) q} \frac{1}{|k|}\left|F_{R}(k \omega)\right|\right) \\
& \leq \frac{n\|p\|_{\infty}}{2 \pi} \sum_{1 \leq s \leq \frac{n}{q}} \frac{1}{s q} \frac{8 q}{R} \log R \\
& =\frac{4 n\|p\|_{\infty}}{\pi R} \log R \sum_{1 \leq s \leq \frac{n}{q}} \frac{1}{s} \\
& \leq \frac{4 n\|p\|_{\infty}}{\pi R} \cdot \log R \cdot \log \left(\frac{n}{q}\right) \leq \frac{4 n\|p\|_{\infty}}{\pi R} \cdot \log R \cdot \log n
\end{aligned}
$$

Hence, using the estimates obtained for $S_{1}(x), S_{2}(x)$ and $S_{3}(x)$, we have

$$
\begin{aligned}
\| \frac{1}{R}( & \left.S_{R} p\right)-\int p \|_{\infty} \leq\left|\sum_{0<|k| \leq n} \hat{p}(k) F_{R}(k \omega) e(k x)\right| \\
& \leq\left|S_{1}(x)\right|+\left|S_{2}(x)\right|+\left|S_{3}(x)\right| \\
& \leq \frac{n\|p\|_{\infty}}{2 \pi R} \cdot[\log (\log R)]^{2} \cdot \log R+\frac{4 n\|p\|_{\infty}}{\pi R} \cdot \log R \cdot[\log (\log R)]^{2} \\
& +\frac{4 n\|p\|_{\infty}}{\pi R} \cdot \log R \cdot \log n \\
& \leq \frac{n\|p\|_{\infty}}{\pi R} \cdot\left(\frac{1}{2 c} \cdot \log R \cdot[\log (\log R)]^{2}+\frac{4 \log R \cdot[\log (\log R)]^{2}}{c}+4(\log R)^{2}\right) \\
& \leq \frac{n\|p\|_{\infty}}{\pi R} \cdot\left(\frac{1}{2 c} \cdot(\log R)^{3}+\frac{4}{c} \cdot(\log R)^{3}+\frac{4}{c} \cdot(\log R)^{3}\right) \\
& \leq \frac{12}{c}\|p\|_{\infty} n \cdot \frac{(\log R)^{3}}{\pi R}
\end{aligned}
$$

since $[\log (\log R)] \leq \log R$ and $0<c<1$. This completes the proof. 
Theorem 4.5.5. Let $u \in C^{\alpha}(\mathbb{T})$, where $0<\alpha<1$ and let $\omega \in \mathbb{T}$. Assume that $\omega$ satisfies the Diophantine condition (4.2). There exists a universal constant $K$ such that for all $R \geq 1$,

$$
\left\|\frac{1}{R}\left(S_{R} u\right)-\int_{\mathbb{T}} u\right\|_{\infty} \leq \frac{K}{c} \frac{(\log R)^{3}}{R^{\frac{\alpha}{\alpha+1}}}\|u\|_{\infty}
$$

where $\left(S_{R} u\right)(x)=u(x)+u(x+\omega)+\cdots+u(x+(R-1) \omega)$.

Proof. Take $n=R^{\frac{1}{\alpha+1}}$.

By Theorem 4.5.1, there exists a universal constant $C<\infty$ and there exists a trigonometric polynomial $p$ of degree $n$ such that

$$
\|u-p\|_{\infty}<C n^{-\alpha}
$$

Hence, applying Lemma 4.5.4, we have that

$$
\begin{aligned}
\| \frac{1}{R}( & \left.S_{R} u\right)-\int u\left\|_{\infty} \leq\right\| \frac{1}{R}\left(S_{R} u\right)-\frac{1}{R}\left(S_{R} p\right)+\frac{1}{R}\left(S_{R} p\right)-\int p+\int p-\int u \|_{\infty} \\
& \leq\left\|\frac{1}{R}\left[S_{R}(u-p)\right]\right\|_{\infty}+\left\|\frac{1}{R}\left(S_{R} p\right)-\int p\right\|_{\infty}+\left\|\int(p-u)\right\|_{\infty} \\
& \leq \frac{1}{R} \sum_{j=0}^{R-1}\|u(x+j \omega)-p(x+j \omega)\|_{\infty}+\frac{12}{c} \cdot\|p\|_{\infty} n \cdot \frac{(\log R)^{3}}{\pi R}+\int\|(p-u)\|_{\infty} \\
& \leq \frac{2 C}{n^{\alpha}}+\frac{12}{c}\|p\|_{\infty} n \cdot \frac{(\log R)^{3}}{\pi R} \\
& \lesssim \frac{2 C}{n^{\alpha}}+\frac{12}{c}\|u\|_{\infty} n \cdot \frac{(\log R)^{3}}{\pi R} \\
& =\frac{1}{R^{\frac{\alpha}{\alpha+1}}}\left(2 C+\frac{12}{c}\|u\|_{\infty} \cdot \frac{(\log R)^{3}}{\pi}\right) \\
& \leq \frac{K}{c} \cdot \frac{(\log R)^{3}}{R^{\frac{\alpha}{\alpha+1}}}\|u\|_{\infty}
\end{aligned}
$$

where $K=12 C$. Hence, we obtain the conclusion that we were looking for.

\section{6}

\section{Related problems}

Let us end with some natural questions related to the main result proved in this chapter.

1. Is the Diophantine condition strictly necessary? Can one consider weaker arithmetic conditions (e.g. Brjuno)? 
2. What happens when $\omega$ is say, the golden ratio (thus satisfying a very strong Diophantine condition), do the estimates get better?

3. What happens in the opposite context, that of a Liouville number $\omega$, does the result fail to hold?

4. Is it possible to obtain a better rate of convergence in Theorem 4.1.1? What is the optimal rate?

5. What if instead of a Hölder observable we consider any continuous observable $u$, can we derive a rate of convergence of the Birkhoff sums that depends explicitly on the modulus of continuity of $u$ ?

6. Are results of this type valid for other uniquely ergodic systems, such as the higher dimensional torus translation or the skew-transltion?

We intend to answer some of the questions above in future works. 


\section{Bibliography}

[1] H. Poincaré. Sur le problème des trois corps et les équations de la dynamique, Acta Mathematica, Vol. 13, 1890.

[2] C. Carathéodory. Über den Wiederkehrsatz von Poincaré, Sitz. Ber. Preuss. Akad. Wiss, Berlin, 1919, pp. 580-584.

[3] G.D. Birkhoff. Proof of the ergodic theorem. Proc. Nat. Acad. Sci. USA, 17 (1931), pp. 656-660.

[4] M. Viana and K. Oliveira. Foundations of ergodic theory, Cambridge Studies in Advanced Mathematics, 2016.

[5] J.F.C. Kingman. The ergodic theory of subadditive stochastic processes, J. Royal Stat. Soc., Vol. B30, 1968, pp. 499-510.

[6] A. Furman. On the multiplicative ergodic theorem for uniquely ergodic systems, Ann. Inst. H. Poincaré Probab. Statist., Vol. 33, 1997, pp. 797-815.

[7] S. Jitomirskaya and R. Mavi. Continuity of the Measure of the Spectrum for Quasiperiodic Schrodinger Operators with Rough Potentials, Communications in Mathematical Physics, Vol. 325.2, 2014, pp. 585-601.

[8] E.M. Stein and R. Shakarchi. Fourier Analysis: An Introduction, Princeton University Press, 2003.

[9] T. Tao. Lecture notes for the honors real analysis course, University of California, Los Angeles, 2003. Available at: http: //www.math.ucla.edu/ $\sim$ tao/resource/general/131bh.1.03s/.

[10] Y. Katznelson and B. Weiss. A simple proof of some ergodic theorems, Israel Journal of Mathematics, Vol. 42, Issue 4, 1982, pp. 291-296.

[11] J. L. Kelley. General Topology, Graduate Texts in Mathematics, Springer New York, 1975. 
[12] S. Lang. Introduction to Diophantine approximations, SpringerVerlag, New York, second edition, 1995.

[13] Y. Katznelson. An Introduction To Harmonic Analysis, Cambridge Mathematical Library, Cambridge University Press, 2004.

[14] L. Kuipers and H. Niederreiter. Uniform distribution of sequences, Wiley-Interscience Publication (John Wiley \& Sons), New York, 1974.

[15] P. Duarte and S. Klein. Continuity of the Lyapunov Exponents of Linear Cocycles, Publicações Matemáticas, IMPA, 2017.

[16] O. Sarig. Lecture Notes on Ergodic Theory, Weizmann Institute of Science, 2009. Available at: http://www.weizmann.ac.il/math/sarigo/ sites/math.sarigo/files/uploads/ergodicnotes.pdf.

[17] M. Viana. Lectures on Lyapunov Exponents, Cambridge Studies in Advanced Mathematics, 2014. 